Journal of the Operations Research

Society of Japan

Vol. 34, No. 1, March 1991

\title{
区間係数を有する多目的線形計画問題に対する 非支配解の概念
}

\author{
乾口雅弘 久米靖文 \\ 大阪府立大学
}

(受理 1989 年 3 月 2 日; 再受理 1990 年 8 月 23 日)

和文概要 本研究では, 区間係数を有する多目的線形計画問題に対する解の概念として, 四 つの非支配解を提案する.屯ず，目的空間上の支配関係を定義し，乙れを用いて决定空間上の支 配関係を構成する.この際, 目的関数が点対集合写像であることから, 可能, 必然などの様相概 念を導入する.得られた决定空間上の支配関係の性質を調べ，四つの非支配解を定義する.次に， 四つの非支配解の性質を調へ，各非支配解が各非支配端点の山結合で表されるととを示す.また， Bitran により提案されている二つの有効解が, 四つの非支配解に含まれているととを示す.

\section{1.はじめに}

線形計画問題を定式化するとき,係数などのパラメータは専門家の経験·知識により一つの 確定值として定められてきた.しかし, 定めるべきパラメータ自体が変動したり,専門家のパラ メータに対する経験·知識が不明確である場合がある.てのよらな場合, 各パラメータを一つの 確定値として定めたのでは，現実問題のモデルとしては不十分である。このよ5な不明確さに対 処する一つの方法として, 感度分析手法やパラメトリック線形計画法が考えられてきた. 従来, てれらの方法では，一つの不明確なパラメータしか扱うととができなかった. 近年，ての欠点に 対処するため, 制約条件の右辺の值および目的関数の係数における複数の不明確なパラメータ を同時に扱ら方法が提案されている [9] [10] [30] [31] [33].

パラメータ自体が変動するときには, パラメータが従ら確率分布を定め, 確率計画問題とし ても定式化されてきた.しかし，データが少な過ぎたり，観測するてとができないため,パラメー タが従う確率分布を推定するととが困難である場合がある。また，データが十分あっても，社会 環境や経済状勢などのような再現性のない要因に依存してパラメータ值が定まる場合は, 過去 のデータから推定された確率分布を用いても現在の問題に対して必ずしも努当であるとはいえ ない.したがって, パラメータ自体が変動する場合にも, 常に確率計画問題として扱うととがで きるとは限らない。

パラメータに対する専門家の経験·知識が不明確であっても, パラメータのとりらる範囲を 定められる場合や, パラメータが従ら確率分布が推定できないときでも, 専門家の経験·知識に より変動しらる範囲を定められる場合がある。このような場合, パラメータを実数の集合で制限 して扱ら方法が考えられる.てのよらな観点から, パラメータを凸集合で制限する Inexact 線形 計画法 1) [1] [8] [30],各パラメータを区間で制限する区間線形計画法 [2] [13] [17] [31], 各パラ メータをファジィ数で制限する可能性線形計画法 [3] [4] [6] [11] [12] [14] [15] [19] [20] [22] [28] [32] [33] などが研究されている.とれらの方法では, 制約条件の右辺の值, 目的関数の係数 
のみならず,制約条件式の係数の不明確さをも考慮できる.同時に複数の不明確なパラメータを 扱える Multiparametric 線形計画法においても,パラメータを集合で制限するもの [9] [10] も る.

てれらの方法では,パラメータの不明確さを反映して現実問題をモデル化している.そのた め, 定式化された問題仕ill-posed な問題となる.しかし，モデルにおける解の合理性あるいは罗 当性を議論せずに適当に解を選べばよいとはいえない. 適当に解を選ぶのであれば, 不明碓さを 反映してモデル化する必要はない.すなわち，てれらの方法では, 解のモデルにおける合理性, 㚣 当性を追求するととは重要である.

複数の目的を取り扱ら多目的線形計画問題に対しては, 感度分析手法やパラメトリック手法 は质とんど提案されていないが, パラメータを区間で制限する区間多目的線形計画法 [2] [13], パ ラメータをファジィ数で制限する可能性多目的線形計画法 [11] [12] [15] [19] [25]〜 [28] が提案 されている.これらの方法では, 問題の複雑さのため, 意思決定者の選好や目的, 制約に対する希 求水準を引を出すことにより取り扱われてをを [11]〜 [13]. 近年, より客観的な立場から合理的 な解を求めるため, 有効解 (パレート解, 非劣解とも呼ばれる) の概念の拡張がなされている [2] [15] [19] [25] [28].

Bitran [2] は, 有効解の概念を素直に拡張し, 目的関数の係数が区間で制限された多目的線 形計画問題に対して，2 種類の有效解が定義できるととを指摘している.それらは，区間で制限 された少なくとも一組のパラメータ值に対して有效となる解と，区間で制限されたすべてのパ ラメ一タ值に対して有効となる解である. 各区間が係数のとりらる範甩, 寸なわち, 係数の可能 性を示していると考えると, てれらはそれぞれ, 可能的な有効解, 必然的な有効解とみなすとと ができる。

必然的有効解は，与えられた区間内のいかなる值が実現しても,通常の多目的線形計画問題 の意味で有効な解となる.一方, 可能的有効解仕, 奏現值が与えられた区間内であっても, 必ずし も有効解になるとは限らない.したがって，必然的有效解の方がより堅実的な解である。しかし， 必然的有效解は常に存在するとは限らない. 可能的有效解は, 実行可能集合が有界で空でなけれ ば, 必ず存在する. 可能的有効解と必然的有効解には, 上述のよらな短所と長所がある.

本研究では, Bitran とは異なった方法で, 区間多目的線形計画問題の 4 種類の解の概念を提 案し, その 2 種類のものが可能的有效解と必然的有效解の中間的なものであるてとを示す.とて で重要なととは，区間が係数の可能性を表しているということである.したがって，係数を制限 している区間は様相論理 [18] そおける可能世界に対応し, 可能, 必然などの様相概念を用いて解 を定義しなければならない。

通常の多目的計画問題に対して, Yu [36] は有效解の拡張である非支配解を提案している.非 支配解は, 解と解との支配関係を表す支配錐を用いて定義される.との支配錐が非正象限 ${ }^{2)}$ K一 致するとを, 非支配解は有效解に一致する.非支配解では, 支配関係が重要な役割をする.

Bitran は有効性の概念を直接拡張したが，本研究では，支配錐が非負象限である支配関係を 拡張するととにより,区間多目的線形計画問題の解の概念を定義する.すべての区間が通常の実 数に退化するとき,てれらの解は通常の多目的線形計画問題の有効解に一致する.こてで定義さ れる解は支配関係を介して構成されるので, 非支配解と呼ぶことにする.

以下では，まず，支配関係を拡張し，その性質を調べる. 次に，拡張された支配関係を用いて 非支配解を定義し, その性質を調べ, 幾何学的な特徵および, 各非支配解が各非支配端点の凸結 合で表されることを明らかにする.最後に，Bitran による二つの有效解との関係を論じ，それら が四つの非支配解に含まれているととを示す。 


\section{2. 支配関係の拡張とその性質}

\section{1 問題設定}

条件 $\mathbf{x} \in F \subseteq \mathbf{R}^{n}$ のもとで, $p$ 個の線形目的関数

$$
\sum_{j=1}^{n} c_{i j} x_{j}, i=1,2, \cdots, p
$$

を最大化する多目的計画問題を, $p \times n$ 行列 $C=\left(c_{i j}\right)$ をちんて,

$$
\operatorname{Max}\{C \mathbf{x} / \mathbf{x} \in F\}
$$

と表すととにする.特に, $F$ が

$$
F=\left\{\mathbf{x} \in \mathbf{R}^{n} / A \mathbf{x}=\mathbf{b}, \mathbf{x} \geqq 0\right\}
$$

と書けるとを，(2.2) 式を多目的線形計画問題と呼ぶ. ただし， $A$ は $m \times n$ 行列, $\mathbf{b}=\left(b_{1}, b_{2}, \cdots\right.$, $\left.b_{m}\right)^{t}$ である.

本研究では, 目的関数の係数が区間で制限された次の多目的線形計画問題を考元る.

$$
\operatorname{Max}\{\Phi \mathbf{x} / \mathbf{x} \in F\}
$$

ただし, $p \times n$ 行列 $C$ の $(i, j)$ 成分を $c_{i j}$ とするとを，

$$
\Phi=\left\{C / \ell_{i j} \leq c_{i j} \leq u_{i j}, i=1,2, \cdots, p, j=1,2, \cdots, n\right\}
$$

である.すなわち， $\Phi$ は各成分が有界な閉区間 $\left[\ell_{i j}, u_{i j}\right]$ で表された $p \times n$ 行列と考えるととがで きる. 各区間 $\left[\ell_{i j}, u_{i j}\right]$ 係数 $c_{i j}$ のとりらる值の範囲を示し, (2.5) 式から明らかなように, 各区 間 $\left[\ell_{i j}, u_{i j}\right]$ 間に相互関係がない ${ }^{3)}$. また, $F$ は $(2.3)$ 式で定義される.

(2.4) 式は, 各目的関数の係数が区間で制限されるので, 通常の多目的線形計画問題の解の概 念をそのまま適用するととができない. 本研究では, (2.4) 式に対する解の概念として, 四つの非 支配解を提案する.なお, 本研究では, (2.4) 式を区間多目的線形計画問題と呼ぶことにする.

\section{2 目的空間上の支配関係}

一般に, 各目的関数の值により, 解の優劣が定められる場合が多い.そとで, 本研究では, 目 的空間上に支配関係が与えられていると仮定し，ての支配関係から，決定空間上の支配関係を導 き出す。

\section{[定義 1]（目的空間上の支配関係）}

目的空間 $\left(\mathbf{R}^{p}\right)$ 上の支配関係 $D$ を

$$
\chi_{D}\left(\mathbf{y}^{1}, \mathbf{y}^{2}\right)=\left\{\begin{array}{l}
1 ; \mathbf{y}^{1} \geqq \mathbf{y}^{2} \\
0 ; \text { その他 }
\end{array}\right.
$$

と定義する.ただし $, \mathbf{y}^{1}, \mathbf{y}^{2} \in \mathbf{R}^{P}$ で， $\chi_{D}$ は $D$ の特性関数である. $\chi_{D}\left(\mathbf{y}^{1}, \mathbf{y}^{2}\right)=1$ であるとも， $\mathbf{y}^{1} \geqq \mathbf{y}^{2}$ であり, $\mathbf{y}^{1}$ が $\mathbf{y}^{2}$ を支配する $\left(\mathbf{y}^{2}\right.$ が $\mathbf{y}^{1}$ 亿支配される) ととを表す.

以後, 記述の簡単のため, 述語および論理記号へ(連言, かつ), V(選言，または)，（否定，で ない $), \rightarrow($ 含意, ならば $), \leftrightarrow\left(\right.$ 同等, 等しい) を用いて表現する. $\mathbf{y}^{1}$ が $\mathbf{y}^{2}$ を支配するととを二項 
述語 $D\left(\mathbf{y}^{1}, \mathbf{y}^{2}\right)$ を用いて表す. また $\Gamma \Rightarrow \Delta$ は $\Delta$ が $\Gamma$ からの論理的㷌結である( $\Gamma$ から $\Delta$ が導 ける)ととを示し,$\Gamma \Leftrightarrow \Delta は \Gamma \Rightarrow \Delta$ かつ $\Delta \Rightarrow \Gamma$ であるととを示す [18].

定義 1 の支配関係は, $Y u[36]$ の支配錐 $\Lambda(\mathbf{y})$ を用いて,

$$
D\left(\mathbf{y}^{1}, \mathbf{y}^{2}\right) \Leftrightarrow \mathbf{y}^{2} \in \mathbf{y}^{1}+\Lambda\left(\mathbf{y}^{1}\right)
$$

と表せる.ただし，

$$
\Lambda(\mathbf{y}) \triangleq \Lambda \leqq=\left\{\lambda \in \mathbf{R}^{P} / \lambda \leqq 0\right\}
$$

であり,

$$
\mathbf{y}^{1}+\Lambda\left(\mathbf{y}^{1}\right)=\left\{\mathbf{y}^{1}+\lambda / \lambda \in \Lambda\left(\mathbf{y}^{1}\right)\right\}
$$

と定義される.すなわち，定義 1 の支配関係は, 支配錐 $\Lambda(\mathbf{y})$ が非正象限と一致する場合の支配 関係である。

明らかに，定義 1 の支配関係は $D\left(\mathbf{y}^{1}, \mathbf{y}^{1}\right)$ が成立し，反射的である.そてで，支配関係 $D$ を 用いて非反射的な強支配関係 $D^{s}$ を次のように定義する.

[定義 2]（目的空間上の強支配関係）

目的空間上の強支配関係 $D^{s}$ を次のよう火定義する。

$$
D^{s}\left(\mathbf{y}^{1}, \mathbf{y}^{2}\right) \triangleq D\left(\mathbf{y}^{1}, \mathbf{y}^{2}\right) \wedge \sim D\left(\mathbf{y}^{2}, \mathbf{y}^{1}\right)
$$

$D^{s}\left(\mathbf{y}^{1}, \mathbf{y}^{2}\right)$ であるとを, $\mathbf{y}^{1}$ が $\mathbf{y}^{2}$ を強く支配するといら。(2.10) 式より， $\mathbf{y}^{1}$ が $\mathbf{y}^{2}$ を強く支 配するととは， $\mathbf{y}^{1}$ が $\mathbf{y}^{2}$ を支配し，かつ， $\mathbf{y}^{2}$ が $\mathbf{y}^{1}$ を支配しないととを表す.明らかに, 次式が成 立する。

$$
D^{s}\left(\mathbf{y}^{1}, \mathbf{y}^{2}\right) \Leftrightarrow \mathbf{y}^{1} \geq \mathbf{y}^{2}
$$

ただし，

$$
\mathbf{y}^{1} \geq \mathbf{y}^{2} \triangleq \mathbf{y}^{1} \geqq \mathbf{y}^{2} \wedge \mathbf{y}^{1} \neq \mathbf{y}^{2}
$$

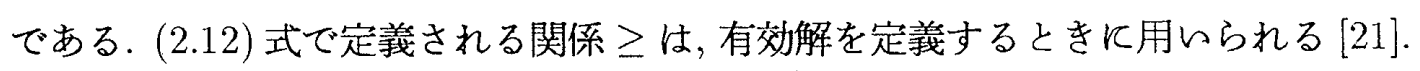

(例 2.1) $\mathbf{x}^{1}=(1,2)^{t}, \mathbf{x}^{2}=(5,0)^{t}, \mathbf{x}^{3}=(2.1)^{t}$,

$$
C^{1}=\left(\begin{array}{ll}
2 & 4 \\
1 & 2
\end{array}\right), C^{2}=\left(\begin{array}{cc}
-2 & -4 \\
1 & 3
\end{array}\right)
$$

とすると, $C^{1} \mathbf{x}^{1}=(10,5)^{t}, C^{1} \mathbf{x}^{2}=(10,5)^{t}, C^{1} \mathbf{x}^{3}=(8,4)^{t}, C^{2} \mathbf{x}^{1}=(-10,7)^{t}, C^{2} \mathbf{x}^{2}$ $=(-10,5)^{t}, C^{2} \mathbf{x}^{3}=(-8,5)^{t}$ となる.とのとを, $C^{1} \mathbf{x}^{1} \geqq C^{1} \mathbf{x}^{2}, C^{1} \mathbf{x}^{2} \geqq C^{1} \mathbf{x}^{1}, C^{1} \mathbf{x}^{1} \geqq C^{1} \mathbf{x}^{3}$, $C^{1} \mathbf{x}^{2} \geqq C^{1} \mathbf{x}^{3}, C^{2} \mathbf{x}^{1} \geqq C^{2} \mathbf{x}^{2}, C^{2} \mathbf{x}^{3} \geqq C^{2} \mathbf{x}^{2}$ が成立するので, $D\left(C^{1} \mathbf{x}^{1}, C^{1} \mathbf{x}^{2}\right), D\left(C^{1} \mathbf{x}^{2}\right.$, $\left.C^{1} \mathbf{x}^{1}\right), D\left(C^{1} \mathbf{x}^{1}, C^{1} \mathbf{x}^{3}\right), D\left(C^{1} \mathbf{x}^{2}, C^{1} \mathbf{x}^{3}\right), D\left(C^{2} \mathbf{x}^{1}, C^{2} \mathbf{x}^{2}\right), D\left(C^{2} \mathbf{x}^{3}, C^{2} \mathbf{x}^{2}\right)$ が成立する。をた， $D\left(C^{1} \mathbf{x}^{1}, C^{1} \mathbf{x}^{2}\right)$ と $D\left(C^{1} \mathbf{x}^{2}, C^{1} \mathbf{x}^{1}\right)$ の両方が成立するので, $D^{s}\left(C^{1} \mathbf{x}^{1}, C^{1} \mathbf{x}^{2}\right)$ \& $D^{s}\left(C^{1} \mathbf{x}^{2}\right.$, $\left.C^{1} \mathbf{x}^{1}\right)$ も成立しないが, $D^{s}\left(C^{1} \mathbf{x}^{1}, C^{1} \mathbf{x}^{3}\right), D^{s}\left(C^{1} \mathbf{x}^{2}, C^{1} \mathbf{x}^{3}\right), D^{s}\left(C^{2} \mathbf{x}^{1}, C^{2} \mathbf{x}^{2}\right), D^{s}\left(C^{2} \mathbf{x}^{3}, C^{2} \mathbf{x}^{2}\right)$ は成立する。

\section{3 決定空間上の支配関係}

通常の多目的線形計画問題では, 各目的関数が点対点写像であるので, 目的空間上の支配関 係から决定空間上の支配関係を唯一に定められる.しかし，(2.4) 式の区間多目的線形計画問題 
では, 各目的関数が点対集合写像である.したがって, 决定変数により定められる目的空間上の 集合をいかに取り扱うかにより，目的空間上の支配関係から種々の决定空間上の支配関係が定 義できる.とてでは，様相論理 [18]で扱われている可能，必然などの様相概念を用いて，决定空 間上の支配関係を定義する.

[定義 3] (可能的支配関係, 必然的支配関係)

可能的支配関係 $\diamond D$, 必然的支配関係 $\square D$ を次のように定義する.

$$
\begin{aligned}
& \diamond D\left(\mathbf{x}^{1}, \mathbf{x}^{2}\right) \triangleq \exists C\left(C \in \Phi \wedge D\left(C \mathbf{x}^{1}, C \mathbf{x}^{2}\right)\right) \\
& \square D\left(\mathbf{x}^{1}, \mathbf{x}^{2}\right) \triangleq \forall C\left(C \in \Phi \rightarrow D\left(C \mathbf{x}^{1}, C \mathbf{x}^{2}\right)\right)
\end{aligned}
$$

(2.13) 式より, $D\left(C \mathbf{x}^{1}, C \mathbf{x}^{2}\right)$ なる $C \in \Phi$ が存在するとをに限り, $\diamond D\left(\mathbf{x}^{1}, \mathbf{x}^{2}\right)$ が成立する. すなわち, $\diamond D\left(\mathbf{x}^{1}, \mathbf{x}^{2}\right)$ が成立するととは, $\mathbf{x}^{1}$ が $\mathbf{x}^{2}$ を支配する場合がある, 言い換えれば, $\mathbf{x}^{1}$ が

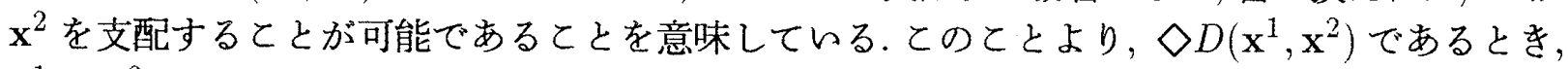

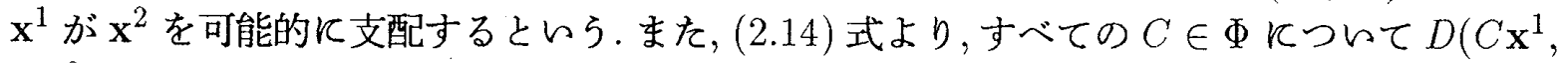
$\left.C \mathbf{x}^{2}\right)$ が成立するときに限り， $\square D\left(\mathbf{x}^{1}, \mathbf{x}^{2}\right)$ が成立する.すなわち， $\square D\left(\mathbf{x}^{1}, \mathbf{x}^{2}\right)$ が成立するとと は， $\mathrm{x}^{1}$ が $\mathrm{x}^{2}$ を常に支配する，言い換えれば， $\mathrm{x}^{1}$ が $\mathrm{x}^{2}$ を支配するととが必然であるととを意味

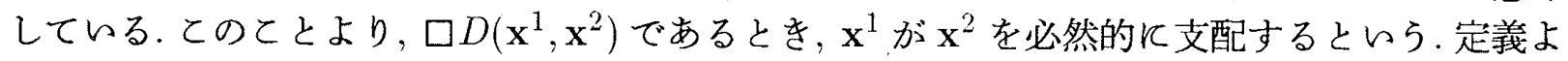
り明らかに，

$$
\square D\left(\mathbf{x}^{1}, \mathbf{x}^{2}\right) \Rightarrow \diamond D\left(\mathbf{x}^{1}, \mathbf{x}^{2}\right)
$$

が成立する。

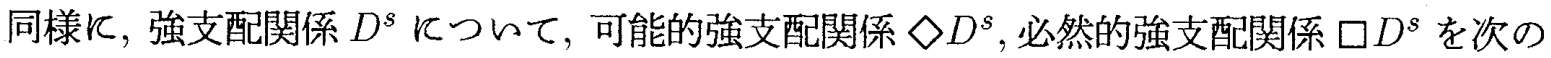
よらに定義する.

[定義 4] (可能的強支配関係, 必然的強支配関係)

可能的強支配関係 $\diamond D^{s}$, 必然的強支配関係 $\square D^{s}$ を次のよう飞定義する.

$$
\begin{aligned}
& \diamond D^{s}\left(\mathbf{x}^{1}, \mathbf{x}^{2}\right) \triangleq \exists C\left(C \in \Phi \wedge D^{s}\left(C \mathbf{x}^{1}, C \mathbf{x}^{2}\right)\right) \\
& \square D^{s}\left(\mathbf{x}^{1}, \mathbf{x}^{2}\right) \triangleq \forall C\left(C \in \Phi \rightarrow D^{s}\left(C \mathbf{x}^{1}, C \mathbf{x}^{2}\right)\right)
\end{aligned}
$$

$\diamond D^{s}\left(\mathbf{x}^{1}, \mathbf{x}^{2}\right)$ であるとき， $\mathbf{x}^{1}$ が $\mathbf{x}^{2}$ を可能的に強支配するといい， $\square D^{s}\left(\mathbf{x}^{1}, \mathbf{x}^{2}\right)$ であると き, $\mathrm{x}^{1}$ が $\mathrm{x}^{2}$ を必然的に強支配するというととにする.明らかに，

$$
\square D^{s}\left(\mathbf{x}^{1}, \mathbf{x}^{2}\right) \Rightarrow \diamond D^{s}\left(\mathbf{x}^{1}, \mathbf{x}^{2}\right)
$$

が成立する。

$\diamond D$ および $\square D$ は， $\diamond D\left(\mathbf{x}^{1}, \mathbf{x}^{1}\right), \square D\left(\mathbf{x}^{1}, \mathbf{x}^{1}\right)$ が成立し, 反射的である.目的空間上の支配 関係 $D$ から強支配関係 $D^{s}$ を構成したよらに, $D, \square D$ から，とれらの重複順列により, 次の 四つの非反射的な関係が得られる.

[定義 5]（弱意強可能的支配関係, 強意強可能的支配関係, 弱意強必然的支配関係, 強意強必然 的支配関係)

决定空間上の 2 点 $\mathbf{x}^{1}, \mathbf{x}^{2}$ そついて, $\diamond D, \square D$ を用いて, 四つの非反射的支配関係を次のよ ら反定義する. 
i) 弱意強可能的支配関係 $0^{3} D$

$$
\text { (.) }{ }^{s} D\left(\mathbf{x}^{1}, \mathbf{x}^{2}\right) \triangleq \diamond D\left(\mathbf{x}^{1}, \mathbf{x}^{2}\right) \wedge \sim \square D\left(\mathbf{x}^{2}, \mathbf{x}^{1}\right)
$$

ii）強意強可能的支配関係 $\widehat{s}^{s} D$

$$
\diamond^{s} D\left(\mathbf{x}^{1}, \mathbf{x}^{2}\right) \triangleq \diamond D\left(\mathbf{x}^{1}, \mathbf{x}^{2}\right) \wedge \sim \diamond D\left(\mathbf{x}^{2}, \mathbf{x}^{1}\right)
$$

iii）弱意強必然的支配関係 四 ${ }^{s} D$

$$
\square^{s} D\left(\mathbf{x}^{1}, \mathbf{x}^{2}\right) \triangleq \square D\left(\mathbf{x}^{1}, \mathbf{x}^{2}\right) \wedge \sim \square D\left(\mathbf{x}^{2}, \mathbf{x}^{1}\right)
$$

iv) 強意強必然的支配関係 $\Xi^{s} D$

$$
\varsigma^{s} D\left(\mathbf{x}^{1}, \mathbf{x}^{2}\right) \triangleq \square D\left(\mathbf{x}^{1}, \mathbf{x}^{2}\right) \wedge \sim \diamond D\left(\mathbf{x}^{2}, \mathbf{x}^{1}\right)
$$

定義 5 より，仝 ${ }^{s} D\left(\mathbf{x}^{1}, \mathbf{x}^{2}\right)$ であるととは， $\mathbf{x}^{1}$ が $\mathrm{x}^{2}$ を可能的に支配し，かつ， $\mathbf{x}^{2}$ が $\mathbf{x}^{1}$ を必

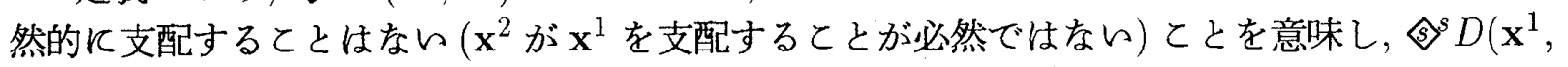
$\left.\mathrm{x}^{2}\right)$ であるととは， $\mathrm{x}^{1}$ が $\mathrm{x}^{2}$ を可能的に支配し，かつ， $\mathrm{x}^{2}$ が $\mathrm{x}^{1}$ を可能的に支配するととはな い $\left(\mathbf{x}^{2}\right.$ が $\mathbf{x}^{1}$ を支配するととが可能ではない)てとを意味する。をた，四 ${ }^{s} D\left(\mathbf{x}^{1}, \mathbf{x}^{2}\right)$ であるとと は， $\mathrm{x}^{1}$ が $\mathrm{x}^{2}$ を必然的に支配し，かつ， $\mathrm{x}^{2}$ が $\mathrm{x}^{1}$ を必然的に支配するととはなんととを意味し， $\square^{s} D\left(\mathbf{x}^{1}, \mathbf{x}^{2}\right)$ であるととは， $\mathbf{x}^{1}$ が $\mathbf{x}^{2}$ を必然的に支配し，かつ， $\mathbf{x}^{2}$ が $\mathbf{x}^{1}$ を可能的に支配すると とはないととを意味する。定義より,明らかに，

$$
\begin{aligned}
& \text { 仓 }^{s} D\left(\mathbf{x}^{1}, \mathbf{x}^{2}\right) \Rightarrow \diamond D\left(\mathbf{x}^{1}, \mathbf{x}^{2}\right) \\
& \text { 今 }^{s} D\left(\mathbf{x}^{1}, \mathbf{x}^{2}\right) \Rightarrow \diamond D\left(\mathbf{x}^{1}, \mathbf{x}^{2}\right)
\end{aligned}
$$

が成立し, (2.12) 式より，

$$
\hat{s}^{s} D\left(\mathbf{x}^{1}, \mathbf{x}^{2}\right) \Rightarrow \text { s. }^{s} D\left(\mathbf{x}^{1}, \mathbf{x}^{2}\right)
$$

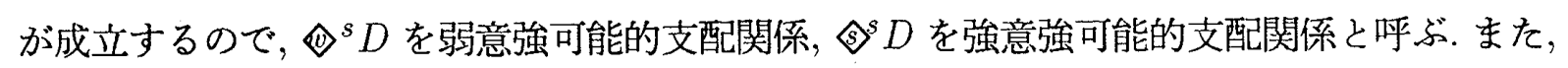

$$
\begin{aligned}
& \varpi^{s} D\left(\mathbf{x}^{1}, \mathbf{x}^{2}\right) \Rightarrow \square D\left(\mathbf{x}^{1}, \mathbf{x}^{2}\right) \\
& \Xi^{s} D\left(\mathbf{x}^{1}, \mathbf{x}^{2}\right) \Rightarrow \square D\left(\mathbf{x}^{1}, \mathbf{x}^{2}\right) \\
& \square^{s} D\left(\mathbf{x}^{1}, \mathbf{x}^{2}\right) \Rightarrow \varpi \varpi^{s} D\left(\mathbf{x}^{1}, \mathbf{x}^{2}\right)
\end{aligned}
$$

が成立するので, 四 ${ }^{s} D$ を弱意強必然的支配関係, $\Xi^{s} D$ を強意強可能的支配関係と呼ぶ.

(例 2.2) (2.4) 式の区間多目的線形計画問題における $\Phi$ および $F$ は非可算集合であるが，とて では，各支配関係を説明するため，いずれも有限個の要素をもつ集合とする.なお，てのように置 き換えても各支配関係の概念は変わらない， $\Phi, F$ を次のように定める.

$$
\begin{aligned}
& \Phi=\left\{C^{1}, C^{2}, C^{3}, C^{4}\right\}, \\
& F=\left\{\mathbf{x}^{1}, \mathbf{x}^{2}, \mathbf{x}^{3}, \mathbf{x}^{4}\right\}, \\
& C^{1}=\left(\begin{array}{ll}
1 & 3 \\
4 & 5
\end{array}\right), C^{2}=\left(\begin{array}{ll}
1 & 3 \\
5 & 3
\end{array}\right), C^{3}=\left(\begin{array}{ll}
3 & 2 \\
4 & 5
\end{array}\right), C^{4}=\left(\begin{array}{ll}
3 & 2 \\
5 & 3
\end{array}\right), \\
& \mathbf{x}^{1}=(3,7)^{t}, \mathbf{x}^{2}=(7,5)^{t}, \mathbf{x}^{3}=(9,1)^{t}, \mathbf{x}^{4}=(0,11)^{t}
\end{aligned}
$$


$\Phi$ は目的関数の実現しらる係数行列の集合を示し, との場合 $C^{1} \sim C^{4}$ の四つの可能性がある. $F$ は実行可能集合であり， $\mathbf{x}^{1} \sim \mathbf{x}^{4}$ の四つの解 (行動) を実行することができる. 目的関数の 各係数行列が実現したときの各解に対する目的関数の值を表 1 に示す.表 1 より, $C^{3} \in \Phi$ つレて $C^{3} \mathbf{x}^{2} \geqq C^{3} \mathbf{x}^{1}\left(D\left(C^{3} \mathbf{x}^{2}, C^{3} \mathbf{x}^{1}\right)\right)$ が成立するので, $C^{3} \in \Phi$ の実現により， $\mathbf{x}^{2}$ は $\mathbf{x}^{1}$ を

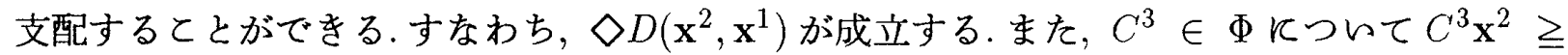
$C^{3} \mathbf{x}^{1}$ かつ $C^{3} \mathbf{x}^{1} \geqq C^{3} \mathbf{x}^{2}\left(D^{s}\left(C^{3} \mathbf{x}^{2}, C^{3} \mathbf{x}^{1}\right)\right)$ が成立するととより, $C^{3} \in \Phi$ の実現により, $\mathbf{x}^{2}$ は $\mathbf{x}^{1}$ を強く支配するてとができる.すなわち, $\diamond D^{s}\left(\mathbf{x}^{2}, \mathbf{x}^{1}\right)$ が成立する.すべての $C^{i} \in \Phi(i=$ $1,2,3,4)$ について $C^{i} \mathbf{x}^{2} \geqq C^{i} \mathbf{x}^{3}$ かつ $C^{i} \mathbf{x}^{3} \geq C^{i} \mathbf{x}^{2}\left(D^{s}\left(C^{i} \mathbf{x}^{2}, C^{i} \mathbf{x}^{3}\right)\right)$ が成立するととより, い ずれの $C^{i} \in \Phi$ が実現しても常に， $\mathbf{x}^{2}$ は $\mathbf{x}^{3}$ を強く支配する、すなわち， $\square D^{s}\left(\mathbf{x}^{2}, \mathbf{x}^{3}\right)$ が成立 する.もちろん, $\square D\left(\mathbf{x}^{2}, \mathbf{x}^{3}\right)$ も成立する. $C^{1} \in \Phi$ につて $C^{1} \mathbf{x}^{1} \geqq C^{1} \mathbf{x}^{3}$ であることより $\diamond D\left(\mathbf{x}^{1}, \mathbf{x}^{3}\right)$ となり,すべての $C^{i} \in \Phi(i=1,2,3,4)$ について $C^{i} \mathbf{x}^{3} \geqq C^{i} \mathbf{x}^{1}$ が成立しないて とより〜 $\square D\left(\mathbf{x}^{3}, \mathbf{x}^{1}\right)\left(\square D\left(\mathbf{x}^{3}, \mathbf{x}^{1}\right)\right.$ が成立しない) となるので, 四 ${ }^{s} D\left(\mathbf{x}^{1}, \mathbf{x}^{3}\right)$ が成立する. しか し, $C^{4} \in \Phi つ 々 て C^{4} \mathbf{x}^{3} \geqq C^{4} \mathbf{x}^{1}$ が成立し, $\diamond D\left(\mathbf{x}^{3}, \mathbf{x}^{1}\right)\left(\sim \diamond D\left(\mathbf{x}^{3}, \mathbf{x}^{1}\right)\right.$ が成立しない)ので， 仓s $D\left(\mathbf{x}^{1}, \mathbf{x}^{3}\right)$ は成立しない. 成立する支配関係を表 2 亿示す.

表 1. 例 2.2 Kおける各解の目的関数の值

\begin{tabular}{|c|c|c|c|c|}
\hline 解 係数行列 & $C^{1}$ & $C^{2}$ & $C^{3}$ & $C^{4}$ \\
\hline $\mathbf{x}^{1}$ & $(24,47)^{t}$ & $(24,36)^{t}$ & $(23,47)^{t}$ & $(23,36)^{t}$ \\
\hline$x^{2}$ & $(22,53)^{t}$ & $(22,50)^{t}$ & $(31,53)^{t}$ & $(31,50)^{t}$ \\
\hline$x^{3}$ & $(12,41)^{t}$ & $(12,48)^{t}$ & $(29,41)^{t}$ & $(29,48)^{t}$ \\
\hline $\mathbf{x}^{4}$ & $(33,55)^{t}$ & $(33,33)^{t}$ & $(22,55)^{t}$ & $(22,33)^{t}$ \\
\hline
\end{tabular}

表 2 . 例 2.2 飞おらて成立する支配関係

\begin{tabular}{|c|c|}
\hline $\mathrm{x}^{1}$ と $\mathrm{x}^{2}$ の支配関係 & $\diamond D\left(\mathbf{x}^{2}, \mathbf{x}^{1}\right), \diamond D^{s}\left(\mathbf{x}^{2}, \mathbf{x}^{1}\right), \Leftrightarrow{ }^{s} D\left(\mathbf{x}^{2}, \mathbf{x}^{1}\right), \diamond \diamond^{s} D\left(\mathbf{x}^{2}, \mathbf{x}^{1}\right)$ \\
\hline $\mathrm{x}^{1}$ と $\mathrm{x}^{3}$ の支配関係 & $\begin{array}{l}\diamond D\left(\mathbf{x}^{1}, \mathbf{x}^{3}\right), \diamond D^{s}\left(\mathbf{x}^{1}, \mathbf{x}^{3}\right), \diamond{ }^{s} D\left(\mathbf{x}^{1}, \mathbf{x}^{3}\right), \diamond D\left(\mathbf{x}^{3}, \mathbf{x}^{1}\right) \\
\diamond D^{s}\left(\mathbf{x}^{3}, \mathbf{x}^{1}\right), \diamond{ }^{s} D\left(\mathbf{x}^{3}, \mathbf{x}^{1}\right)\end{array}$ \\
\hline $\mathrm{x}^{1}$ と $\mathrm{x}^{4}$ の支配関係 & $\begin{array}{l}\diamond D\left(\mathbf{x}^{1}, \mathbf{x}^{4}\right), \diamond D^{s}\left(\mathbf{x}^{1}, \mathbf{x}^{4}\right), \diamond^{s} D\left(\mathbf{x}^{1}, \mathbf{x}^{4}\right), \diamond D\left(\mathbf{x}^{4}, \mathbf{x}^{1}\right) \\
\diamond D^{s}\left(\mathbf{x}^{4}, \mathbf{x}^{1}\right), \otimes^{s} D\left(\mathbf{x}^{4}, \mathbf{x}^{1}\right)\end{array}$ \\
\hline $\mathrm{x}^{2}$ と $\mathrm{x}^{3}$ の支配関係 & $\begin{array}{l}\diamond D\left(\mathbf{x}^{2}, \mathbf{x}^{3}\right), \square D\left(\mathbf{x}^{2}, \mathbf{x}^{3}\right), \diamond D^{s}\left(\mathbf{x}^{2}, \mathbf{x}^{3}\right), \square D^{s}\left(\mathbf{x}^{2}, \mathbf{x}^{3}\right), \\
\diamond^{s} D\left(\mathbf{x}^{2}, \mathbf{x}^{3}\right), \diamond s D\left(\mathbf{x}^{2}, \mathbf{x}^{3}\right), \varpi^{s} D\left(\mathbf{x}^{2}, \mathbf{x}^{3}\right), \square^{s} D\left(\mathbf{x}^{2}, \mathbf{x}^{3}\right)\end{array}$ \\
\hline $\mathrm{x}^{2}$ と $\mathrm{x}^{4}$ の支配関係 & $\begin{array}{l}\diamond D\left(\mathbf{x}^{2}, \mathbf{x}^{4}\right), \diamond D^{s}\left(\mathbf{x}^{2}, \mathbf{x}^{4}\right), \diamond{ }^{s} D\left(\mathbf{x}^{2}, \mathbf{x}^{4}\right), \diamond D\left(\mathbf{x}^{4}, \mathbf{x}^{2}\right) \\
\diamond D^{s}\left(\mathbf{x}^{4}, \mathbf{x}^{2}\right), \otimes^{s} D\left(\mathbf{x}^{4}, \mathbf{x}^{2}\right)\end{array}$ \\
\hline $\mathrm{x}^{3}$ と $\mathrm{x}^{4}$ の支配関係 & $\begin{array}{l}\diamond D\left(\mathbf{x}^{3}, \mathbf{x}^{4}\right), \diamond D^{s}\left(\mathbf{x}^{3}, \mathbf{x}^{4}\right), \otimes^{s} D\left(\mathbf{x}^{3}, \mathbf{x}^{4}\right), \diamond D\left(\mathbf{x}^{4}, \mathbf{x}^{3}\right) \\
\diamond D^{s}\left(\mathbf{x}^{4}, \mathbf{x}^{3}\right), \otimes^{s} D\left(\mathbf{x}^{4}, \mathbf{x}^{3}\right)\end{array}$ \\
\hline その他 & $\begin{array}{l}\diamond D\left(\mathbf{x}^{1}, \mathbf{x}^{1}\right), \square D\left(\mathbf{x}^{1}, \mathbf{x}^{1}\right), \diamond D\left(\mathbf{x}^{2}, \mathbf{x}^{2}\right), \square D\left(\mathbf{x}^{2}, \mathbf{x}^{2}\right) \\
\diamond D\left(\mathbf{x}^{3}, \mathbf{x}^{3}\right), \square D\left(\mathbf{x}^{3}, \mathbf{x}^{3}\right), \diamond D\left(\mathbf{x}^{4}, \mathbf{x}^{4}\right), \square D\left(\mathbf{x}^{4}, \mathbf{x}^{4}\right)\end{array}$ \\
\hline
\end{tabular}




\section{4 性質}

こてでは, 主として 2.3 で定義された決定空間上の非反射的な六つの支配関係 $\diamond D^{s}, \square D^{s}$,

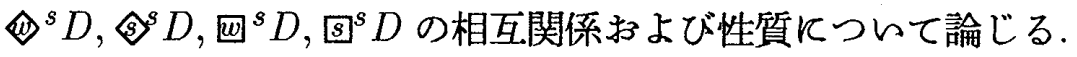

[命題 1] 次式が成立する.

$$
\begin{aligned}
& \varpi^{s} D\left(\mathbf{x}^{1}, \mathbf{x}^{2}\right) \Rightarrow \otimes^{s} D\left(\mathbf{x}^{1}, \mathbf{x}^{2}\right) \\
& \square^{s} D\left(\mathbf{x}^{1}, \mathbf{x}^{2}\right) \Rightarrow \diamond^{s} D\left(\mathbf{x}^{1}, \mathbf{x}^{2}\right)
\end{aligned}
$$

（証明）定義 5 および.(2.12) 式より明らかである.

[命題 2] 次式が成立する.

$$
\begin{gathered}
\diamond D^{s}\left(\mathbf{x}^{1}, \mathbf{x}^{2}\right) \Leftrightarrow \otimes^{s} D\left(\mathbf{x}^{1}, \mathbf{x}^{2}\right) \\
\square D^{s}\left(\mathbf{x}^{1}, \mathbf{x}^{2}\right) \Leftrightarrow \Theta^{s} D\left(\mathbf{x}^{1}, \mathbf{x}^{2}\right) \\
\square D^{s}\left(\mathbf{x}^{1}, \mathbf{x}^{2}\right) \Leftrightarrow \Xi^{s} D\left(\mathbf{x}^{1}, \mathbf{x}^{2}\right) \wedge \varpi^{s} D\left(\mathbf{x}^{1}, \mathbf{x}^{2}\right)
\end{gathered}
$$

（証明）定義 $2 \sim 5$ より，

$$
\begin{aligned}
& \diamond D^{s}\left(\mathbf{x}^{1}, \mathbf{x}^{2}\right) \\
& =\exists C\left(C \in \Phi \wedge D\left(C \mathbf{x}^{1}, C \mathbf{x}^{2}\right) \wedge \sim D\left(C \mathbf{x}^{2}, C \mathbf{x}^{1}\right)\right) \\
& \square D^{s}\left(\mathbf{x}^{1}, \mathbf{x}^{2}\right) \\
& =\forall C\left(C \in \Phi \rightarrow D\left(C \mathbf{x}^{1}, C \mathbf{x}^{2}\right) \wedge \sim D\left(C \mathbf{x}^{2}, C \mathbf{x}^{1}\right)\right) \\
& \text { (0) }{ }^{s} D\left(\mathbf{x}^{1}, \mathbf{x}^{2}\right) \\
& =\exists C\left(C \in \Phi \wedge D\left(C \mathbf{x}^{1}, C \mathbf{x}^{2}\right)\right) \\
& \wedge \sim \forall C\left(C \in \Phi \rightarrow D\left(C \mathbf{x}^{2}, C \mathbf{x}^{1}\right)\right) \\
& =\exists C\left(C \in \Phi \wedge D\left(C \mathbf{x}^{1}, C \mathbf{x}^{2}\right)\right) \\
& \wedge \exists C\left(C \in \Phi \wedge \sim D\left(C \mathbf{x}^{2}, C \mathbf{x}^{1}\right)\right) \\
& \text { (s) }{ }^{s} D\left(\mathbf{x}^{1}, \mathbf{x}^{2}\right) \\
& =\forall C\left(C \in \Phi \rightarrow D\left(C \mathbf{x}^{1}, C \mathbf{x}^{2}\right)\right) \\
& \wedge \sim \exists C\left(C \in \Phi \wedge D\left(C \mathbf{x}^{2}, C \mathbf{x}^{1}\right)\right) \\
& =\forall C\left(C \in \Phi \rightarrow D\left(C \mathbf{x}^{1}, C \mathbf{x}^{2}\right)\right) \\
& \wedge \forall C\left(C \in \Phi \rightarrow \sim D\left(C \mathrm{x}^{2}, C \mathrm{x}^{1}\right)\right) \\
& =\forall C\left(C \in \Phi \rightarrow D\left(C \mathbf{x}^{1}, C \mathbf{x}^{2}\right) \wedge \sim D\left(C \mathbf{x}^{2}, C \mathbf{x}^{1}\right)\right)
\end{aligned}
$$

となり ${ }^{4)}, \diamond D^{s}\left(\mathbf{x}^{1}, \mathbf{x}^{2}\right) \Rightarrow$ 仝 $^{s} D\left(\mathbf{x}^{1}, \mathbf{x}^{2}\right)$ および $(2.32)$ 式は明らかである。 ڤ $^{s} D\left(\mathbf{x}^{1}, \mathbf{x}^{2}\right) \Rightarrow$ $\diamond D^{s}\left(\mathbf{x}^{1}, \mathbf{x}^{2}\right)$ について証明する。四 ${ }^{s} D\left(\mathbf{x}^{1}, \mathbf{x}^{2}\right)$ が成立すると仮定すると， $D\left(C^{1} \mathbf{x}^{2}, C^{1} \mathbf{x}^{1}\right)$ な る $C^{1} \in \Phi$ が存在する. $\left(C^{1}\right)_{i}$. を $C^{1}$ の $i$ 番目の行とすると, 定義 1 より, $\left(C^{1}\right)_{i^{*}} \cdot \mathbf{x}^{1}>\left(C^{1}\right)_{i^{*}} \cdot \mathbf{x}^{2}$ なる $i^{*} \in\{1,2, \cdots, p\}$ が存在する.一方, 众 ${ }^{s} D\left(\mathbf{x}^{1}, \mathbf{x}^{2}\right)$ が成立するので, $D\left(C^{2} \mathbf{x}^{1}, C^{2} \mathbf{x}^{2}\right)$ なる $C^{2} \in \Phi$ が存在する. 定義 1 より, 任意の $i \in\{1,2, \cdots, p\}$ 凡つんて, $\left(C^{2}\right)_{i} \cdot \mathbf{x}^{1} \geqq\left(C^{2}\right)_{i} \cdot \mathbf{x}^{2}$ が成 立する. $C^{2}$ の $i^{*}$ 番目の行 $\left(C^{2}\right)_{i^{*}}$. を $\left(C^{1}\right)_{i^{*}}$. K置を換えを行列を $C^{3}$ とすると, $C^{3} \mathbf{x}^{1} \geq C^{3} \mathbf{x}^{2}$ となる。いま，区間で表された $\Phi$ 各成分間に相互関係がないので, $C^{3} \in \Phi$ となる。したがっ $\tau,(2.11)$ 式より, $C^{3} \in \Phi \wedge D^{s}\left(C^{3} \mathbf{x}^{1}, C^{3} \mathbf{x}^{2}\right)$ が成立し, $\diamond D^{s}\left(\mathbf{x}^{1}, \mathbf{x}^{2}\right)$ が成立する. 


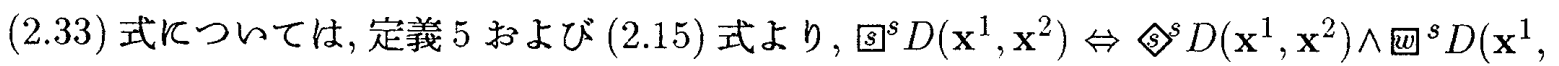
$\left.\mathrm{x}^{2}\right)$ が成立するととおよび, $(2.32)$ 式より明らかである。

(証明終)

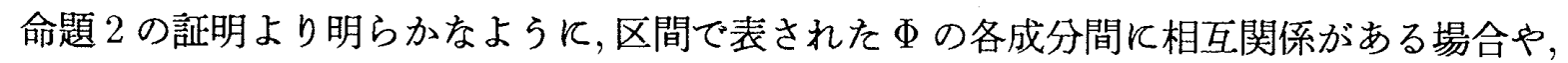
支配関係として定義 1 以外のものを用いる場合には, 必ずしも (2.31) 式が成立するとは限らな

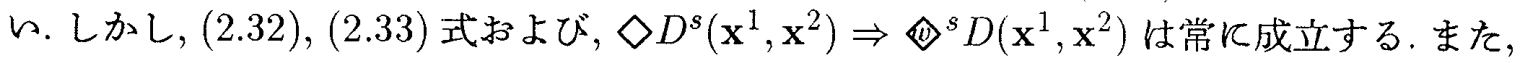

$$
\diamond^{s} D\left(\mathbf{x}^{1}, \mathbf{x}^{2}\right) \vee \varpi^{s} D\left(\mathbf{x}^{1}, \mathbf{x}^{2}\right) \Rightarrow \diamond D^{s}\left(\mathbf{x}^{1}, \mathbf{x}^{2}\right)
$$

は成立するが, 逆は成立しない ${ }^{5)}$.

以上の議論より, 非反射的な六つの支配関係 $\diamond D^{s}, \square D^{s}, \otimes^{s} D, \diamond s s^{s} D,{ }^{s} D, \Theta^{s} D$ の相互関 係を図示すると, 図 1 のようになる.図 1 より, $\square D^{s}$ およ゙ $s^{s} D$ が最も強く, $\diamond D^{s}$ およ゙ 创Dが最も弱いてとがわかる。

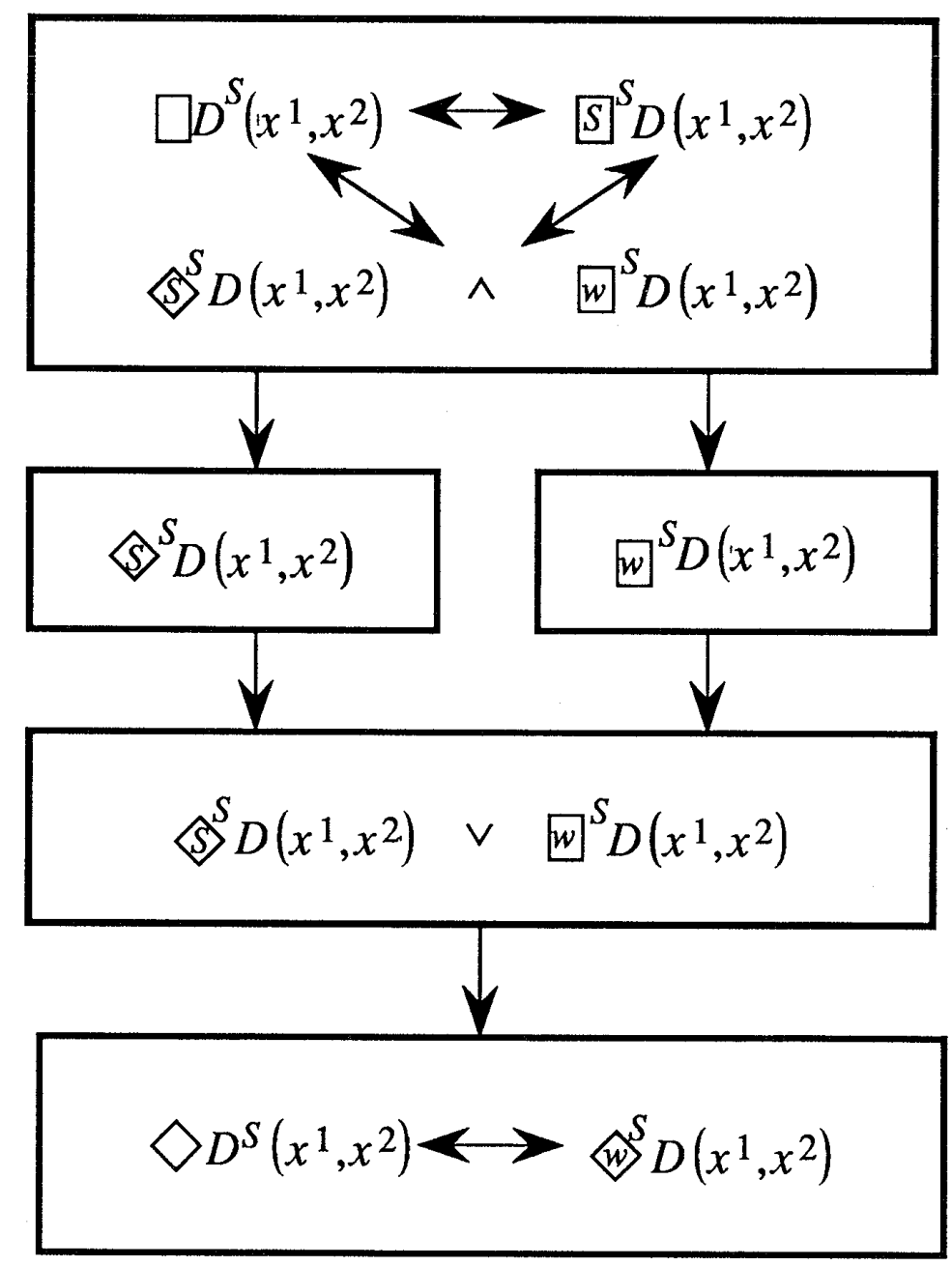

図 1 非反射的な六つの支配関係の相互関係

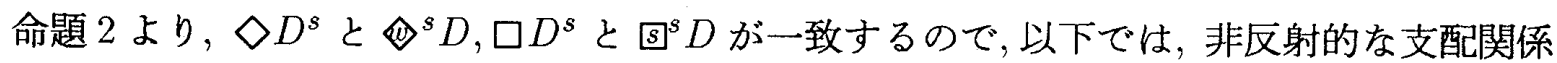

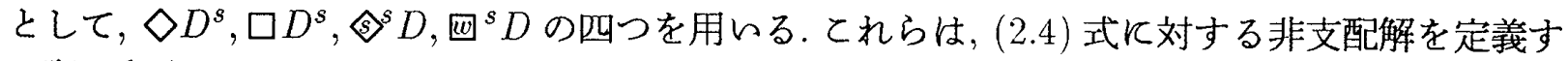
る際, 重要となる。

ここで,

$$
P \triangleq\{1,2, \cdots, p\}
$$




$$
\begin{gathered}
Q\left(\mathbf{x}^{1}, \mathbf{x}^{2}\right) \triangleq\left\{\mathbf{c} \in \mathbf{R}^{n} /<\mathbf{c}, \mathbf{x}^{1}-\mathbf{x}^{2}>\geqq 0\right\} \\
\Phi_{i} \triangleq\left\{C_{i} . \in \mathbf{R}^{n} / C \in \Phi\right\}
\end{gathered}
$$

と定義する.ただし， $<\mathbf{x}^{1}, \mathbf{x}^{2}>$ は $\mathbf{x}^{1}$ と $\mathbf{x}^{2}$ の内積を表し， $C_{i}$ は $C$ の $i$ 番目の行である.との とき, 次の命題が成立する.

[命題 3] 次式が成立する.

$$
\begin{aligned}
& \diamond D^{s}\left(\mathbf{x}^{1}, \mathbf{x}^{2}\right) \\
& \Leftrightarrow \exists i\left(i \in P \wedge \operatorname{Int} Q\left(\mathbf{x}^{1}, \mathbf{x}^{2}\right) \cap \Phi_{i . \neq \phi}\right) \\
& \wedge \forall i\left(i \in P \rightarrow Q\left(\mathbf{x}^{1}, \mathbf{x}^{2}\right) \cap \Phi_{i .} \neq \phi\right) \\
& \square D^{s}\left(\mathbf{x}^{1}, \mathbf{x}^{2}\right) \\
& \Leftrightarrow \exists i\left(i \in P \wedge \Phi_{i} \subseteq \operatorname{Int} Q\left(\mathbf{x}^{1}, \mathbf{x}^{2}\right)\right) \\
& \wedge \forall i\left(i \in P \rightarrow \Phi_{i} \subseteq Q\left(\mathbf{x}^{1}, \mathbf{x}^{2}\right)\right) \\
& \diamond^{s} D\left(\mathbf{x}^{1}, \mathbf{x}^{2}\right) \\
& \Leftrightarrow \exists i\left(i \in P \wedge \Phi_{i} \subseteq \operatorname{Int} Q\left(\mathbf{x}^{1}, \mathbf{x}^{2}\right)\right) \\
& \wedge \forall i\left(i \in P \rightarrow Q\left(\mathbf{x}^{1}, \mathbf{x}^{2}\right) \cap \Phi_{i} \neq \phi\right) \\
& \bigotimes^{s} D\left(\mathbf{x}^{1}, \mathbf{x}^{2}\right) \\
& \Leftrightarrow \exists i\left(i \in P \wedge \operatorname{Int} Q\left(\mathbf{x}^{1}, \mathbf{x}^{2}\right) \cap \Phi_{i . \neq \phi} \neq \phi\right. \\
& \wedge \forall i\left(i \in P \rightarrow \Phi_{i} \subseteq Q\left(\mathbf{x}^{1}, \mathbf{x}^{2}\right)\right)
\end{aligned}
$$

ただし, Int $Q\left(\mathbf{x}^{1}, \mathbf{x}^{2}\right)$ は $Q\left(\mathbf{x}^{1}, \mathbf{x}^{2}\right)$ の内点の集合である.

（証明）定義および $\Phi$ の各成分間に相互関係がないととより明らかである.

命題 3 壮，各支配関係の幾何学的な特徵を表している.

(例 2.4) $\mathbf{x}^{1}=(5,4)^{t}, \mathbf{x}^{2}=(3,2)^{t}$ とし, $\ell_{11}=-3, u_{11}=-2, \ell_{12}=2, u_{12}=3, \ell_{21}=$ $-1.5, u_{21}=-0.5, \ell_{22}=-0.5, u_{22}=1, \ell_{31}=0, u_{31}=2, \ell_{32}=-3, u_{32}=-2$ とする. との とき, $Q\left(\mathbf{x}^{1}, \mathbf{x}^{2}\right), \Phi_{i} .(i=1,2,3)$ を四示すると, 困 2.a のようになる. $1 \in P=\{1,2,3\}$ K対して

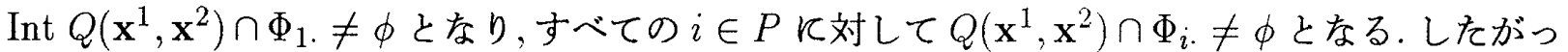

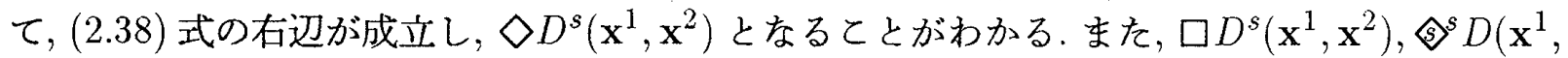
$\left.\mathbf{x}^{2}\right)$, 四 ${ }^{s} D\left(\mathbf{x}^{1}, \mathbf{x}^{2}\right)$ は成立しない.

(例 2.5) $\mathbf{x}^{1}=(5,4)^{t}, \mathbf{x}^{2}=(3,2)^{t}$ とし, $\ell_{11}=-2, u_{11}=-1, \ell_{12}=2, u_{12}=4, \ell_{21}=1, u_{21}=$ $2, \ell_{21}=-1, u_{21}=0, \ell_{31}=2, u_{31}=3.5, \ell_{32}=-2, u_{32}=-1.5$ とすると, 図 $2 . \mathrm{b}$ より, $1 \in P=$ $\{1,2,3\}$ に対して Int $Q\left(\mathbf{x}^{1}, \mathbf{x}^{2}\right) \cap \Phi_{1} . \neq \phi$ となり,すべての $i \in P$ 亿対して $\Phi_{i} \subseteq Q\left(\mathbf{x}^{1}, \mathbf{x}^{2}\right)$ となる.したがって, (2.38), (2.41) 式の右辺が成立し, $\diamond D^{s}\left(\mathbf{x}^{1}, \mathbf{x}^{2}\right)$, 四 ${ }^{s} D\left(\mathbf{x}^{1}, \mathbf{x}^{2}\right)$ となる. また， $\square D^{s}\left(\mathbf{x}^{1}, \mathbf{x}^{2}\right)$, 仓s $D\left(\mathbf{x}^{1}, \mathbf{x}^{2}\right)$ は成立しない。

(例 2.6) $\mathrm{x}^{1}=(5,4)^{t}, \mathrm{x}^{2}=(3,2)^{t}$ とし, $\ell_{11}=-3, u_{11}=-1, \ell_{12}=1, u_{12}=2, \ell_{21}=2, u_{21}=$ $3, \ell_{22}=-1, u_{22}=1, \ell_{31}=1, u_{31}=1.5, \ell_{32}=-3, u_{32}=-1.5$ とすると, $Q\left(\mathbf{x}^{1}, \mathbf{x}^{2}\right), \Phi_{i} .(i=$ $1,2,3)$ は図 2.c のよ5になる.図 2.cより, $2 \in P=\{1,2,3\}$ に対して $\Phi_{2} . \subseteq \operatorname{Int} Q\left(\mathbf{x}^{1}, \mathbf{x}^{2}\right)$ とな 
り,すべての $i \in P$ K対して $Q\left(\mathbf{x}^{1}, \mathbf{x}^{2}\right) \cap \Phi_{i} \neq \neq \phi$ となる.したがって, (2.38), (2.40) 式の右辺が 成立し, $\diamond D^{s}\left(\mathbf{x}^{1}, \mathbf{x}^{2}\right), \diamond s\left(\mathbf{x}^{1}, \mathbf{x}^{2}\right)$ となる. $\square D^{s}\left(\mathbf{x}^{1}, \mathbf{x}^{2}\right)$, 四 ${ }^{s} D\left(\mathbf{x}^{1}, \mathbf{x}^{2}\right)$ 代成立しない。

(例 2.7) $\mathbf{x}^{1}=(5,4)^{t}, \mathbf{x}^{2}=(3,2)^{t}$ とし, $\ell_{11}=-2, u_{11}=-1, \ell_{12}=2, u_{12}=2.5, \ell_{21}=$ $1, u_{21}=2, \ell_{22}=2, u_{22}=3, \ell_{31}=2.5, u_{31}=4, \ell_{32}=-2, u_{32}=-1$ とすると, 図 $2 . \mathrm{d}$ より, $2 \in$ $P=\{1,2,3\}$ に対して $\Phi_{2} \subseteq$ Int $Q\left(\mathbf{x}^{1}, \mathbf{x}^{2}\right)$ となり,すべのて $i \in P$ K対して $\Phi_{i} \subseteq Q\left(\mathbf{x}^{1}, \mathbf{x}^{2}\right)$ となる.したがって, $(2.38) \sim(2.41)$ 式の右辺がすべて成立し, $\diamond D^{s}\left(\mathbf{x}^{1}, \mathbf{x}^{2}\right), \square D^{s}\left(\mathbf{x}^{1}, \mathbf{x}^{2}\right)$, $\widehat{\diamond}^{s} D\left(\mathbf{x}^{1}, \mathbf{x}^{2}\right)$, 四 ${ }^{s} D\left(\mathbf{x}^{1}, \mathbf{x}^{2}\right)$ となる。

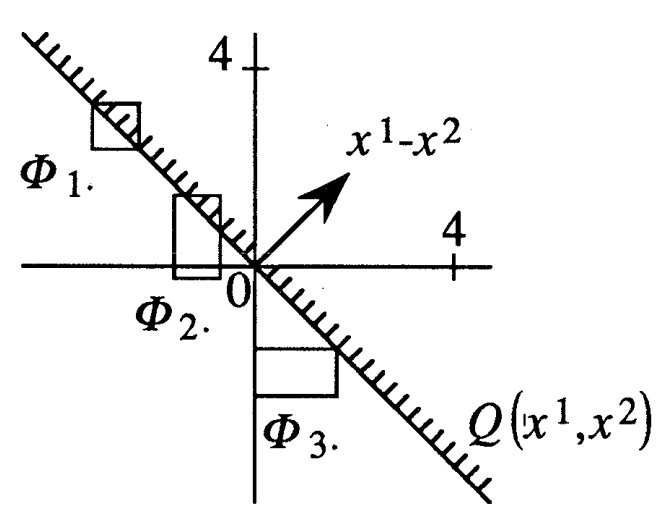

a. 例 2.4

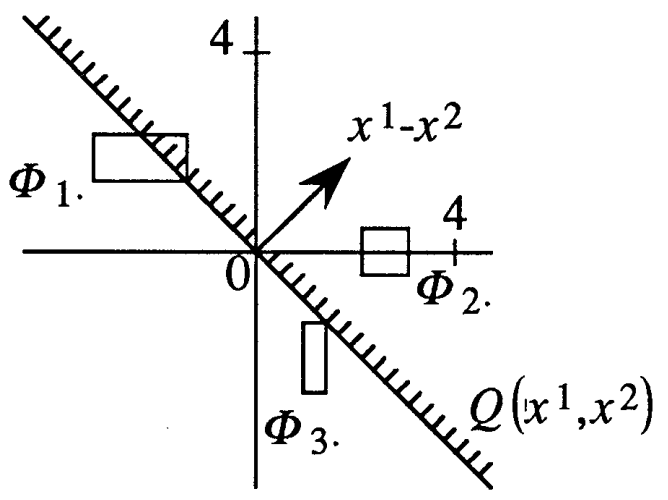

c. 例 2.6

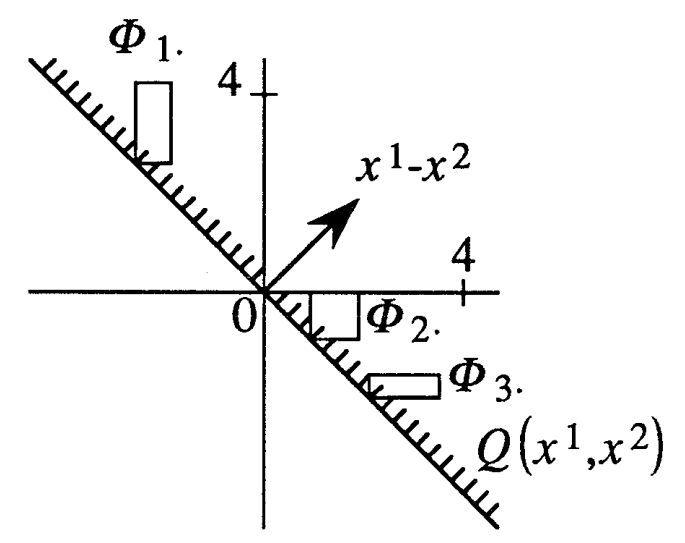

b. 例 2.5

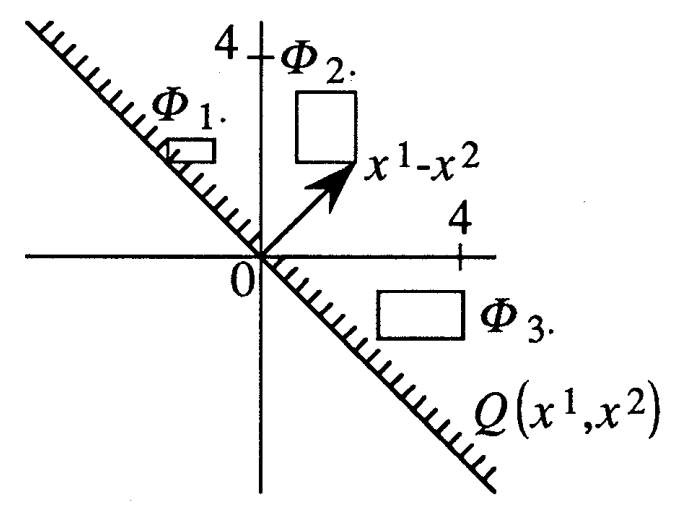

d. 例 2.7

図 2 非反射的な支配関係

[命題 4] 次式が成立する.

$$
\begin{gathered}
\diamond D\left(\mathbf{x}^{1}, \mathbf{x}^{2}\right) \Leftrightarrow \exists C\left(C \in M \wedge D\left(C \mathbf{x}^{1}, C \mathbf{x}^{2}\right)\right) \\
\square D\left(\mathbf{x}^{1}, \mathbf{x}^{2}\right) \Leftrightarrow \forall C\left(C \in M \rightarrow D\left(C \mathbf{x}^{1}, C \mathbf{x}^{2}\right)\right) \\
\diamond D^{s}\left(\mathbf{x}^{1}, \mathbf{x}^{2}\right) \Leftrightarrow \exists C\left(C \in M \wedge D^{s}\left(C \mathbf{x}^{1}, C \mathbf{x}^{2}\right)\right) \\
\square D^{s}\left(\mathbf{x}^{1}, \mathbf{x}^{2}\right) \Leftrightarrow \forall C\left(C \in M \rightarrow D^{s}\left(C \mathbf{x}^{1}, C \mathbf{x}^{2}\right)\right)
\end{gathered}
$$

ただし，

$$
M=\left\{C / C_{\cdot j}=L \cdot j \text { または } C_{\cdot j}=U_{\cdot j}, j=1,2, \cdot, n\right\}
$$


であり, $C$ は $p \times n$ 行列 $, L, U$ はそれぞれ, $(i, j)$ 成分を $\ell_{i j}, u_{i j}$ とする $p \times n$ 行列である. また， $C . j$ は $C$ 番目の列を表している.

(証明) (2.42) 式を証明する. $M \subseteq \Phi$ おび定義 3 より，

$$
\exists C\left(C \in M \wedge D\left(C \mathbf{x}^{1}, C \mathbf{x}^{2}\right)\right) \Rightarrow \diamond D\left(\mathbf{x}^{1}, \mathbf{x}^{2}\right)
$$

は明らかである.したがって，ての逆を示せばよい。 $\diamond D\left(\mathbf{x}^{1}, \mathbf{x}^{2}\right)$ が成立すると仮定すると, 定義 1,3 より,$C^{1} \mathbf{x}^{1} \geqq C^{1} \mathbf{x}^{2}$, つまり,$C^{1}\left(\mathbf{x}^{1}-\mathbf{x}^{2}\right) \geqq 0$ なる $C^{1} \in \Phi$ が存在する. $\mathbf{x}^{1}-\mathbf{x}^{2}$ の $j$ 成分 $\left(\mathbf{x}^{1}-\mathbf{x}^{2}\right)_{j}$ と記し, 行列 $C^{2}$ を

$$
C_{\cdot j}^{2}=\left\{\begin{array}{l}
U_{\cdot j} ;\left(\mathbf{x}^{1}-\mathbf{x}^{2}\right)_{j} \geqq 0 \\
L_{\cdot j} ;\left(\mathbf{x}^{1}-\mathbf{x}^{2}\right)_{j}<0
\end{array}\right.
$$

と定義すると, $C^{2}\left(\mathbf{x}^{1}-\mathbf{x}^{2}\right) \geqq C^{1}\left(\mathbf{x}^{1}-\mathbf{x}^{2}\right) \geqq 0, C^{2} \in M$ が成立する.ゆえに, $C^{2} \in M, C^{2} \mathbf{x}^{1} \geqq$ $C^{2} \mathbf{x}^{2}$ が成立し, $\exists C\left(C \in M \wedge D\left(C \mathbf{x}^{1}, C \mathbf{x}^{2}\right)\right)$ が成立する.

他も，同様に証明するととができる.

(証明終)

命題 4 は，支配関係 $\diamond D, \square D, \diamond D^{s}, \square D^{s}, \widehat{s}^{s} D$, 四 ${ }^{s} D$ の成立を議論するとき，係数が区間 の両端の值をとるとをだけを考えれば十分であるてとを示している.したがって, 非可算集合 $\Phi$ のたかだか $2^{n}$ 個の要素をもつ部分集合 $M$ についてのみ, 支配関係 $D, D^{s}$ の成立を議論すれば

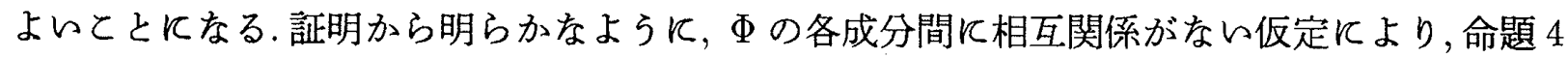
が成立する.さらに，相互関係がならとんら仮定のもとでは, 係数のとりらる值の領域が区間で なくても, 最大值 $u_{i j}$ および最小值 $\ell i j$ が存在しさえすれば, 命題 4 恃成立する.

\section{3. 非支配解の定義と性質}

\section{1 非支配解の定義}

决定空間上の非反射的な四つの支配関係 $\diamond D^{s}, \square D^{s},\left(\diamond^{s} D\right.$, 四 ${ }^{s} D$ を用々て, 四つ ${ }^{6)}$ の非支 配解が定義でをる。

[定義 6] (非支配必然解, 非支配可能解, 弱意非支配必然解, 強意非支配可能解)

区間多目的線形計画問題 (2.4) 式に対して, 次の四つの非支配解が定義できる.

i） $\mathbf{x}^{*}$ が非支配必然解であるととを $\square N D\left(\mathbf{x}^{*}\right)$ と記し，次のように定義する.

$$
\begin{aligned}
& \square N D\left(\mathbf{x}^{*}\right) \\
& \triangleq \mathbf{x}^{*} \in F \wedge \sim \exists \mathbf{x}\left(\mathbf{x} \in F \wedge \diamond D^{s}\left(\mathbf{x}, \mathbf{x}^{*}\right)\right)
\end{aligned}
$$

ii） $\mathbf{x}^{*}$ が非支配可能解であるととを $\diamond N D\left(\mathbf{x}^{*}\right)$ と記し，次のよらに定義する.

$$
\begin{aligned}
& \diamond N D\left(\mathbf{x}^{*}\right) \\
& \triangleq \mathbf{x}^{*} \in F \wedge \sim \exists \mathbf{x}\left(\mathbf{x} \in F \wedge \square D^{s}\left(\mathbf{x}, \mathbf{x}^{*}\right)\right)
\end{aligned}
$$

iii） $\mathbf{x}^{*}$ が弱意非支配必然解であるととを四 $N D\left(\mathbf{x}^{*}\right)$ と記し，次のように定義する。

$$
\begin{aligned}
& \text { 四D }\left(\mathbf{x}^{*}\right) \\
& \triangleq \mathbf{x}^{*} \in F \wedge \sim \exists \mathbf{x}\left(\mathbf{x} \in F \wedge\left(\S^{s} D\left(\mathbf{x}, \mathbf{x}^{*}\right)\right)\right.
\end{aligned}
$$


iv) $\mathbf{x}^{*}$ が強意非支配可能解であるととを仓 $N D\left(\mathbf{x}^{*}\right)$ と記し, 次のように定義する.

$$
\begin{aligned}
& \triangleq N D\left(\mathbf{x}^{*}\right) \\
& \triangleq \mathbf{x}^{*} \in F \wedge \sim \exists \mathbf{x}\left(\mathbf{x} \in F \wedge \varpi^{s} D\left(\mathbf{x}, \mathbf{x}^{*}\right)\right)
\end{aligned}
$$

定義 6 より， $\square N D\left(\mathbf{x}^{*}\right)$ が成立するととは， $\mathbf{x}^{*}$ を可能的に強支配する $\mathbf{x} \in F$ が存在しない， すなわち,すべての $\mathrm{x} \in F$ K対して $\mathrm{x}^{*}$ が強支配されないてとが必然であるととを意味する。乙 の意味において, 非支配必然解と呼ぶ. $\diamond N D\left(\mathbf{x}^{*}\right)$ が成立するととは, $\mathbf{x}^{*}$ を必然的に強支配す る $\mathbf{x} \in F$ が存在しない，すなわち，すべての $\mathbf{x} \in F$ K対して $\mathbf{x}^{*}$ が強支配されないてとが可能 であるととを意味する。ての意味において, 非支配可能解と呼ぶ. また, 四 $N D, \diamond N D$ 仗, 強意強 可能的支配関係 今s $D$, 弱意強必然的支配関係 四 ${ }^{s} D$ から定義されるととおよび, 定義氏より, 強 弱関係, 可能一必然が逆転するととにより, 弱意非支配必然解, 強意非支配可能解と呼ぶ.

特 $K, \ell_{i j}=u_{i j}, i=1,2, \cdots, p, j=1,2, \cdots, n$ すなわち, (2.4) 式が通常の多目的線形計画 問題に退化するとをには; 定義 1 より, 定義 6 の非支配解はすべて有効解に一致する. をた，図 1 に示す相互関係より明らかに，

$$
\begin{aligned}
& \square N D\left(\mathbf{x}^{*}\right) \Rightarrow 凹 N D\left(\mathbf{x}^{*}\right) \\
& \square N D\left(\mathbf{x}^{*}\right) \Rightarrow \S N D\left(\mathbf{x}^{*}\right) \\
& \text { 四 } N D\left(\mathbf{x}^{*}\right) \Rightarrow \diamond N D\left(\mathbf{x}^{*}\right) \\
& \diamond N D\left(\mathbf{x}^{*}\right) \Rightarrow \diamond N D\left(\mathbf{x}^{*}\right)
\end{aligned}
$$

が成立する.とれらの関係を図 3 に示す.

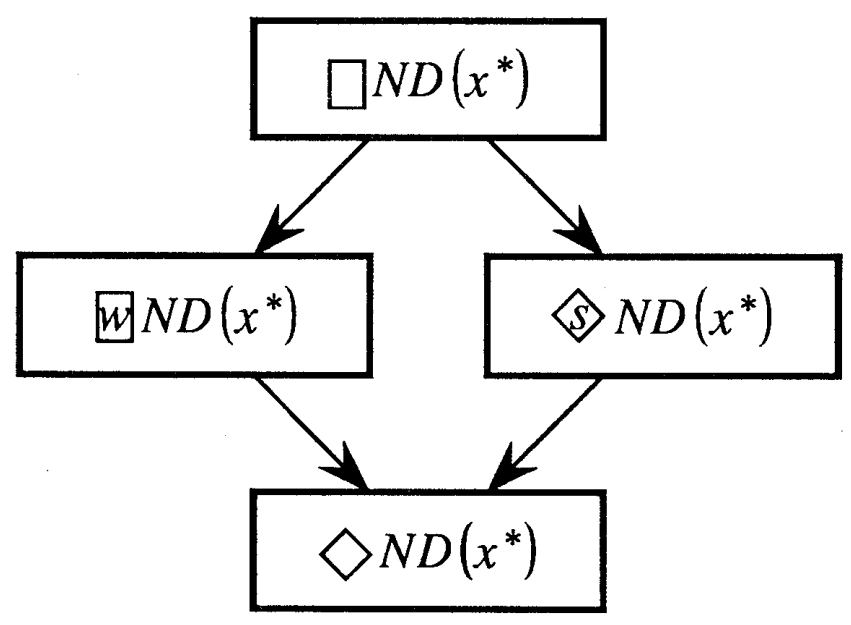

困 3 四つの非支配解の相互関係

(例 3.1) $\Phi, F$ が有限個の要素をもつ例 2.2 の場合を考える. $\mathbf{x}^{1} \in F$ 亿対して，表 $2 よ り ，$ 四 ${ }^{s} D\left(\mathbf{x}^{i}, \mathbf{x}^{1}\right)$ なる $\mathbf{x}^{i} \in F$ が存在しない.したがって, (3.4) 式の右辺が成立し, $\diamond N D\left(\mathbf{x}^{1}\right)$ とな る. 寸なわち, $\mathbf{x}^{1}$ は強意非支配可能解である. 図 3 より, $\mathbf{x}^{1}$ 注非支配可能解でもある $(\diamond N D$

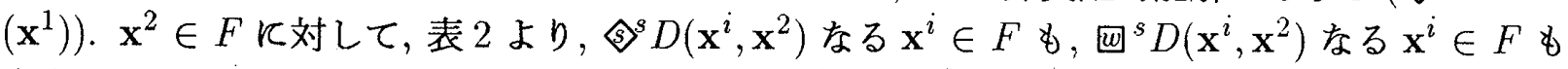
存在しない.したがって, (3.3), (3.4) 式の右辺が成立し, $\mathbf{x}^{2}$ 恃弱意非支配必然解 $\left(\right.$ 回 $\left.N D\left(\mathbf{x}^{2}\right)\right)$ お よび強意非支配可能解 $\left(\bigotimes N D\left(\mathrm{x}^{2}\right)\right)$ であるてとがわかる.図 3 より, $\mathrm{x}^{2}$ 仕非支配可能解 $(\diamond N D$ $\left.\left(\mathbf{x}^{2}\right)\right)$ でもある. $\mathbf{x}^{3} \in F$ K対して, 表 2 より, $\square D^{s}\left(\mathbf{x}^{i}, \mathbf{x}^{3}\right)$ なる $\mathbf{x}^{i} \in F$ が存在する $\left(\square D^{s}\right.$ 
$\left.\left(\mathbf{x}^{2}, \mathbf{x}^{3}\right)\right)$. したがって, (3.2) 式が成立せず, $\mathbf{x}^{3}$ 壮非支配可能解ではない $\left(\sim \diamond N D\left(\mathbf{x}^{3}\right)\right)$. 図 3 よ り, $\mathrm{x}^{3}$ は非支配必然解でも, 弱意非支配必然解でも, 強意非支配可能解でもない. 同様にして, $\mathrm{x}^{4}$ が弱意非支配必然解, 強意非支配可能解および非支配可能解であり, 非支配必然解でないととが わかる。

\section{2 諸性質}

ととで, $\mathbf{x} \in F$ が非支配解でないといら述語をそれぞれ，次のように定義する.

$$
\begin{aligned}
& \neg \square N D(\mathbf{x}) \triangleq \mathbf{x} \in F \wedge \sim \square N D(\mathbf{x}) \\
& \neg \diamond N D(\mathbf{x}) \triangleq \mathbf{x} \in F \wedge \sim \diamond N D(\mathbf{x}) \\
& \neg \text { 四D }(\mathbf{x}) \triangleq \mathbf{x} \in F \wedge \sim \text { 四D }(\mathbf{x}) \\
& \neg \diamond N D(\mathbf{x}) \triangleq \mathbf{x} \in F \wedge \sim \diamond N D(\mathbf{x})
\end{aligned}
$$

また，

$$
\begin{aligned}
& {\left[\mathbf{x}^{1}, \mathbf{x}^{2}\right] \triangleq\left\{\mathbf{x} / \mathbf{x}=\lambda \mathbf{x}^{1}+(1-\lambda) \mathbf{x}^{2}, 0 \leqq \lambda \leqq 1\right\}} \\
& \left(\mathbf{x}^{1}, \mathbf{x}^{2}\right] \triangleq\left\{\mathbf{x} / \mathbf{x}=\lambda \mathbf{x}^{1}+(1-\lambda) \mathbf{x}^{2}, 0 \leqq \lambda<1\right\} \\
& \left(\mathbf{x}^{1}, \mathbf{x}^{2}\right) \triangleq\left\{\mathbf{x} / \mathbf{x}=\lambda \mathbf{x}^{1}+(1-\lambda) \mathbf{x}^{2}, 0<\lambda<1\right\}
\end{aligned}
$$

と定義すると, 以下の命題が成立する.

[命題 5] 次式が成立する.

$$
\begin{aligned}
\mathbf{x}^{1} \in F & \wedge \neg \square N D\left(\mathbf{x}^{2}\right) \wedge \mathbf{x} \in\left(\mathbf{x}^{1}, \mathbf{x}^{2}\right] \\
& \Rightarrow \neg \square N D(\mathbf{x}) \\
\mathbf{x}^{1} \in F & \wedge \neg \diamond N D\left(\mathbf{x}^{2}\right) \wedge \mathbf{x} \in\left(\mathbf{x}^{1}, \mathbf{x}^{2}\right] \\
& \Rightarrow \neg \diamond N D(\mathbf{x}) \\
\mathbf{x}^{1} \in F & \wedge \neg \emptyset N D\left(\mathbf{x}^{2}\right) \wedge \mathbf{x} \in\left(\mathbf{x}^{1}, \mathbf{x}^{2}\right] \\
& \Rightarrow \neg \varpi N D(\mathbf{x}) \\
\mathbf{x}^{1} \in F & \wedge \neg \S N D\left(\mathbf{x}^{2}\right) \wedge \mathbf{x} \in\left(\mathbf{x}^{1}, \mathbf{x}^{2}\right] \\
& \Rightarrow \neg \diamond N D(\mathbf{x})
\end{aligned}
$$

(証明) すべて同様に証明できるので，とてで㤬，(3.18) 式を証明する. $\mathbf{x} \in\left(\mathbf{x}^{1}, \mathrm{x}^{2}\right]$ より, $\bar{\lambda} \in$ $[0,1)$ が存在して, $\mathbf{x}=\bar{\lambda} \mathbf{x}^{1}+(1-\bar{\lambda}) \mathbf{x}^{2}$ と表せる. $\mathbf{x}^{1}, \mathbf{x}^{2} \in F$ および $F$ の凸性より, $\mathbf{x} \in F$ と なる。䧃 $N D\left(\mathbf{x}^{2}\right)$ が成立するので, 定義 $1,5,6$ より, $C^{2} \overline{\mathbf{x}}^{2} \geqq C^{2} \mathbf{x}^{2}$ なる $C^{2} \in \Phi$ が存在し, か つ,すべての $C \in \Phi$ について $C \mathbf{x}^{2} \geqq C \overline{\mathbf{x}}^{2}$ が成立するような $\overline{\mathbf{x}}^{2} \in F$ が存在する.乙たがって， $C^{2} \in \Phi$ につレ,$C^{2}\left(\bar{\lambda} \mathbf{x}^{1}+(1-\bar{\lambda}) \overline{\mathbf{x}}^{2}\right) \geqq C^{2}\left(\bar{\lambda} \mathbf{x}^{1}+(1-\bar{\lambda}) \mathbf{x}^{2}\right)=C^{2} \mathbf{x}$ が成立し，かつ，すへ ての $C \in \Phi$ につて, $C\left(\bar{\lambda} \mathbf{x}^{1}+(1-\bar{\lambda}) \mathbf{x}^{2}\right) \geqq C\left(\bar{\lambda} \mathbf{x}^{1}+(1-\bar{\lambda}) \overline{\mathbf{x}}^{2}\right)$ が成立する. $\mathbf{x}^{1}, \overline{\mathbf{x}}^{2} \in F$ お よび $F$ の凸性より, $\bar{\lambda} \mathbf{x}^{1}+(1-\bar{\lambda}) \overline{\mathbf{x}}^{2} \in F$ となるので, 定義 $1,5,6 よ り ＼sim$ 四 $N D(\mathbf{x})$ が成立す る.ゆえに, 四 $N D(\mathbf{x})$ となる。

(言正明終) 
[命題 6] 次式が成立する.

$$
\begin{aligned}
& \neg \square N D\left(\mathbf{x}^{1}\right) \wedge \neg \square N D\left(\mathbf{x}^{2}\right) \wedge \mathbf{x} \in\left[\mathbf{x}^{1}, \mathbf{x}^{2}\right] \\
& \Rightarrow \neg \square N D(\mathbf{x}) \\
& \neg \diamond N D\left(\mathbf{x}^{1}\right) \wedge \neg \diamond N D\left(\mathbf{x}^{2}\right) \wedge \mathbf{x} \in\left[\mathbf{x}^{1}, \mathbf{x}^{2}\right] \\
& \Rightarrow \neg \diamond N D(\mathbf{x}) \\
& \neg 凹 N D\left(\mathbf{x}^{1}\right) \wedge \neg \emptyset N D\left(\mathbf{x}^{2}\right) \wedge \mathbf{x} \in\left[\mathbf{x}^{1}, \mathbf{x}^{2}\right] \\
& \Rightarrow \neg \text { 四 } N D(\mathbf{x}) \\
& \neg \diamond N D\left(\mathbf{x}^{1}\right) \wedge \neg \diamond N D\left(\mathbf{x}^{2}\right) \wedge \mathbf{x} \in\left[\mathbf{x}^{1}, \mathbf{x}^{2}\right] \\
& \Rightarrow \neg \diamond N D(\mathbf{x})
\end{aligned}
$$

（証明）命題 5 より明らかである.

(証明終)

[命題 7] 次式が成立する.

$$
\begin{aligned}
\mathbf{x}^{1} \in F & \wedge \mathbf{x}^{2} \in F \wedge \mathbf{x}^{0} \in\left(\mathbf{x}^{1}, \mathbf{x}^{2}\right) \\
& \wedge \square N D\left(\mathbf{x}^{0}\right) \wedge \mathbf{x} \in\left[\mathbf{x}^{1}, \mathbf{x}^{2}\right] \Rightarrow \square N D(\mathbf{x}) \\
\mathbf{x}^{1} \in F & \wedge \mathbf{x}^{2} \in F \wedge \mathbf{x}^{0} \in\left(\mathbf{x}^{1}, \mathbf{x}^{2}\right) \\
& \wedge \diamond N D\left(\mathbf{x}^{0}\right) \wedge \mathbf{x} \in\left[\mathbf{x}^{1}, \mathbf{x}^{2}\right] \Rightarrow \diamond N D(\mathbf{x}) \\
\mathbf{x}^{1} \in F & \wedge \mathbf{x}^{2} \in F \wedge \mathbf{x}^{0} \in\left(\mathbf{x}^{1}, \mathbf{x}^{2}\right) \\
& \wedge \varpi N D\left(\mathbf{x}^{0}\right) \wedge \mathbf{x} \in\left[\mathbf{x}^{1}, \mathbf{x}^{2}\right] \Rightarrow \varpi N D(\mathbf{x}) \\
\mathbf{x}^{1} \in F & \wedge \mathbf{x}^{2} \in F \wedge \mathbf{x}^{0} \in\left(\mathbf{x}^{1}, \mathbf{x}^{2}\right) \\
& \wedge \diamond N D\left(\mathbf{x}^{0}\right) \wedge \mathbf{x} \in\left[\mathbf{x}^{1}, \mathbf{x}^{2}\right] \Rightarrow \diamond N D(\mathbf{x})
\end{aligned}
$$

証明法，命題 5 より,通常の多目的線形計画問題の場合 [21] と同様に証明できるので，省略 する.

[命題 8] 次式が成立する.

$$
\begin{aligned}
\mathbf{x}^{1} \in F & \wedge \mathbf{x}^{2} \in F \wedge \mathbf{x}^{0} \in\left(\mathbf{x}^{1}, \mathbf{x}^{2}\right) \\
& \wedge \neg \square N D\left(\mathbf{x}^{0}\right) \wedge \mathbf{x} \in\left(\mathbf{x}^{1}, \mathbf{x}^{2}\right) \Rightarrow \neg \square N D(\mathbf{x}) \\
\mathbf{x}^{1} \in F & \wedge \mathbf{x}^{2} \in F \wedge \mathbf{x}^{0} \in\left(\mathbf{x}^{1}, \mathbf{x}^{2}\right) \\
& \wedge \neg \diamond N D\left(\mathbf{x}^{0}\right) \wedge \mathbf{x} \in\left(\mathbf{x}^{1}, \mathbf{x}^{2}\right) \Rightarrow \neg \diamond N D(\mathbf{x}) \\
\mathbf{x}^{1} \in F & \wedge \mathbf{x}^{2} \in F \wedge \mathbf{x}^{0} \in\left(\mathbf{x}^{1}, \mathbf{x}^{2}\right) \\
& \wedge \neg \emptyset N D\left(\mathbf{x}^{0}\right) \wedge \mathbf{x} \in\left(\mathbf{x}^{1}, \mathbf{x}^{2}\right) \Rightarrow \neg \varpi N D(\mathbf{x}) \\
\mathbf{x}^{1} \in F & \wedge \mathbf{x}^{2} \in F \wedge \mathbf{x}^{0} \in\left(\mathbf{x}^{1}, \mathbf{x}^{2}\right) \\
& \wedge \neg \diamond N D\left(\mathbf{x}^{0}\right) \wedge \mathbf{x} \in\left(\mathbf{x}^{1}, \mathbf{x}^{2}\right) \Rightarrow \neg \diamond N D(\mathbf{x})
\end{aligned}
$$


(証明) $\left(\mathbf{x}^{1}, \mathbf{x}^{2}\right)=\left(\mathbf{x}^{1}, \mathbf{x}^{0}\right] \cup\left(\mathbf{x}^{2}, \mathbf{x}^{0}\right]$ および命題 5 より明らかである.

(証明終)

非支配必然解であるような $F$ の端点を非支配必然端点, 非支配可能解であるような $F$ の端 点を非支配可能端点, 弱意非支配必然解であるような $F$ の端点を弱意非支配必然端点, 強意非 支配可能解であるような $F$ の端点を強意非支配可能端点と呼ぶととにする. 次の定理が成り立 ว.

[定理 1] $F$ が有界であるとき, 次の $(1) \sim(4)$ が成立する.

（1）すべての非支配必然解は, 非支配必然端点の凸結合で表される.

(2) すべての非支配可能解は, 非支配可能端点の凸結合で表される.

(3) すべての弱意非支配必然解は, 弱意非支配必然端点の凸結合で表される.

(4) すべての強意非支配可能解は, 強意非支配可能端点の凸結合で表される.

証明は，命題 5 より，通常の多目的線形計画問題の場合 [21] と同様て証明でをるので, 省略 する.

定理 1 より, $F$ が有界である場合には, 各非支配解が各非支配端点の凸結合で表されるとと がわかる.とのととより，Fが有界である場合，すべての非支配端点を求めるととは，すべての非 支配解を求めるらえで, 重要となる.

[命題 9] 次式が成立する.

$$
\begin{aligned}
& \square \\
& \quad \Leftrightarrow D\left(\mathbf{x}^{*}\right) \\
& \diamond N D\left(\mathbf{x}^{*}\right) \\
& \quad \Leftrightarrow \mathbf{x}^{*} \in F \wedge F \subseteq \mathbf{x}^{*}+\left(\cap_{i \in P} \diamond R_{i} \cup \bigcup_{i \in P} \operatorname{Int} \diamond R_{i}\right) \\
& \text { 冈 } N D\left(\mathbf{x}^{*}\right) \\
& \Leftrightarrow \mathbf{x}^{*} \in F \wedge F \subseteq \mathbf{x}^{*}+\left(\cap_{i \in P} \diamond R_{i} \cup \bigcup_{i \in P} \operatorname{Int} \square R_{i}\right) \\
& \diamond N D\left(\mathbf{x}^{*}\right) \\
& \Leftrightarrow \mathbf{x}^{*} \in F \wedge F \subseteq \mathbf{x}^{*}+\left(\cap_{i \in P} \square R_{i} \cup \bigcup_{i \in P} \operatorname{Int} \diamond R_{i}\right)
\end{aligned}
$$

ただし，

$$
\begin{aligned}
& \square R_{i}=\left\{\mathbf{x} \in \mathbf{R}^{n} / \forall C_{i} .\left(C_{i} \in \Phi_{i} . \rightarrow\left\langle\mathbf{x}, C_{i} .\right\rangle \leqq 0\right)\right\} \\
& \diamond R_{i}=\left\{\mathbf{x} \in \mathbf{R}^{n} / \exists C_{i .}\left(C_{i} \in \Phi_{i .} \wedge\left\langle\mathbf{x}, C_{i} .\right\rangle \leqq 0\right)\right\}
\end{aligned}
$$

である。

(証明) すべて同様に証明できるので，ててでは, (3.35) 式を証明する.命題 3 , 定義 6 より，

$$
\begin{aligned}
\text { (s) } N D\left(\mathbf{x}^{*}\right) \Leftrightarrow \mathbf{x}^{*} \in F \\
\wedge \sim \exists \mathbf{x}\left(\mathbf{x} \in F \wedge \exists i\left(i \in P \wedge \operatorname{Int} Q\left(\mathbf{x}, \mathbf{x}^{*}\right) \cap \Phi_{i} \neq \phi\right)\right.
\end{aligned}
$$




$$
\left.\wedge \forall i\left(i \in P \rightarrow \Phi_{i} \subseteq Q\left(\mathbf{x}, \mathbf{x}^{*}\right)\right)\right)
$$

が成立する.右辺を変形して，

$$
\begin{aligned}
\diamond N D\left(\mathbf{x}^{*}\right) & \Leftrightarrow \mathbf{x}^{*} \in F \\
\wedge \forall \mathbf{x}\left(\mathbf { x } \in F \rightarrow \left(\forall i\left(i \in P \rightarrow \operatorname{Int} Q\left(\mathbf{x}, \mathbf{x}^{*}\right) \cap \Phi_{i} \neq \phi\right)\right.\right. & \\
\vee & \left.\exists i\left(i \in P \wedge \Phi_{i} \cap \bar{Q}\left(\mathbf{x}, \mathbf{x}^{*}\right) \neq \phi\right)\right)
\end{aligned}
$$

となる.ただし, $\bar{Q}\left(\mathbf{x}, \mathbf{x}^{*}\right)$ は $Q\left(\mathbf{x}, \mathbf{x}^{*}\right)$ の補集合である.したがって，

$$
\begin{aligned}
\mathbf{x} \in \mathbf{x}^{*}+\left(\cap_{i \in P} \square R_{i} \cup \bigcup_{i \in P} \operatorname{Int} \diamond R_{i}\right) \\
\Leftrightarrow \forall i\left(i \in P \rightarrow \operatorname{Int} Q\left(\mathbf{x}, \mathbf{x}^{*}\right) \cap \Phi_{i}=\phi\right) \\
\vee \exists i\left(i \in P \wedge \Phi_{i} . \cap \bar{Q}\left(\mathbf{x}, \mathbf{x}^{*}\right) \neq \phi\right)
\end{aligned}
$$

を示せばよい．

$$
\begin{gathered}
\mathbf{x} \in \mathbf{x}^{*}+\left(\cap_{i \in P} \square R_{i} \cup \bigcup_{i \in P} \operatorname{Int} \diamond R_{i}\right) \\
\Leftrightarrow \mathbf{x}-\mathbf{x}^{*} \in \cap_{i \in P} \square R_{i} \vee \mathbf{x}-\mathbf{x}^{*} \in \cup_{i \in P} \operatorname{Int} \diamond R_{i} \\
\Leftrightarrow \forall i\left(i \in P \rightarrow \forall C_{i \cdot}\left(C_{i} \in \Phi_{i} \rightarrow\left\langle\mathbf{x}-\mathbf{x}^{*}, C_{i}\right\rangle \leqq 0\right)\right) \\
\vee \exists i\left(i \in P \wedge \exists C_{i} \cdot\left(C_{i} . \in \Phi_{i} \wedge\left\langle\mathbf{x}-\mathbf{x}^{*}, C_{i}\right\rangle<0\right)\right) \\
\Leftrightarrow \forall i\left(i \in P \rightarrow \operatorname{Int} Q\left(\mathbf{x}, \mathbf{x}^{*}\right) \cap \Phi_{i}=\phi\right) \\
\vee \exists i\left(i \in P \wedge \Phi_{i} \cap \bar{Q}\left(\mathbf{x}, \mathbf{x}^{*}\right) \neq \phi\right)
\end{gathered}
$$

となる。

(証明終)

命題 9 は, 各非支配解の幾何学的な性質を示している.特に, 区間多目的線形計画問題 $(2.4)$ 式がスラック変数を含むとき, 次のようになる。一般性を欠くととなく，

$$
A=\left(\begin{array}{c:c}
A^{1} & I \\
\hdashline & O^{1}
\end{array}\right), \Phi=\left(\Phi^{1}: O^{2}\right)
$$

と表せる.ただし, $I$ は $m_{1}(<m)$ 次の単位行列, $O^{1}, O^{2}$ はそれぞれ, $\left(m-m_{1}\right) \times m_{1}, p \times m_{1}$ の 零行列である.んま，

$$
\begin{aligned}
& F_{1}=\left\{\mathbf{x}^{\prime} \in \mathbf{R}^{n-m_{1}} / A^{1} \mathbf{x}^{\prime} \leqq \mathbf{b}, \mathbf{x}^{\prime} \geqq 0\right\} \\
& \square R_{i}^{1}=\left\{\mathbf{x}^{\prime} \in \mathbf{R}^{n-m_{1}} / \forall C_{i .}^{1}\left(C_{i .}^{1} \in \Phi_{i .}^{1} \rightarrow\left\langle\mathbf{x}^{\prime}, C_{i}^{1}\right\rangle \leqq 0\right)\right\} \\
& \diamond R_{i}^{1}=\left\{\mathbf{x}^{\prime} \in \mathbf{R}^{n-m_{1}} / \exists C_{i .}^{1}\left(C_{i .}^{1} \in \Phi_{i .}^{1} \wedge\left\langle\mathbf{x}^{\prime}, C_{i .}^{1}\right\rangle \leqq 0\right)\right\}
\end{aligned}
$$

とすると, $F_{1}$ は $F$ の $\mathbf{R}^{n-m_{1}}$ への射影であり, $\square R_{i}=\square R_{i}^{1} \times \mathbf{R}^{m_{1}}, \diamond R_{i}=\diamond R_{i}^{1} \times \mathbf{R}^{m_{1}}$ が成 立する. また, 射影 $\psi: F \rightarrow F_{1}$ を考えると, $\psi$ 恃全単射になるので, $(3.32) \sim(3.35)$ 式の右边の

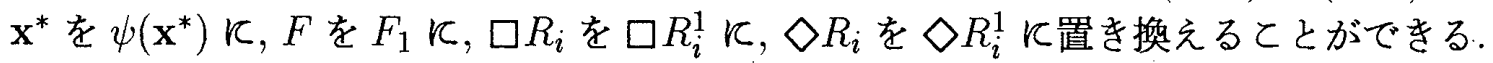

ことで, $p=2, n=4, m=2$ とし,

$$
A=\left(\begin{array}{cccc}
3 & 5 & 1 & 0 \\
1 & 1 & 0 & 1
\end{array}\right), \mathbf{b}=\left(\begin{array}{c}
40 \\
10
\end{array}\right), \mathrm{x}^{*}=(5,5,0,0)^{t}
$$


とした以下の例を考える。

(例 3.2) $\ell_{11}=-3, u_{11}=-1, \ell_{12}=-4, u_{12}=-3, \ell_{13}=u_{13}=\ell_{14}=u_{14}=0, \ell_{21}=$ $4, u_{21}=5, \ell_{22}=2, u_{22}=4, \ell_{23}=u_{23}=\ell_{24}=u_{24}=0$ とする.てのとを, (3.40), (3.41) 式 の $\square R_{i}^{1}, \diamond R_{i}^{1}(i=1,2)$ 恃図 4 亿示すような錐になる.図 4 より, $\left(\diamond R_{1}^{1} \cap \diamond R_{2}^{1}\right) \cup \operatorname{Int} \diamond R_{1}^{1} \cup$ Int $\diamond R_{2}^{1}=\mathbf{R}^{2}$ となり, $\psi\left(\mathbf{x}^{*}\right) \in F_{1}\left(\mathbf{x}^{*} \in F\right)$ が成立し,

$$
F \subseteq \psi\left(\mathbf{x}^{*}\right)+\left(\left(\diamond R_{1}^{1} \cap \diamond R_{2}^{1}\right) \cup \operatorname{Int} \diamond R_{1}^{1} \cup \operatorname{Int} \diamond R_{2}^{1}\right)
$$

も成立するので, $\mathbf{x}^{*}$ は非支配可能解 $\left(\diamond N D\left(\mathbf{x}^{*}\right)\right)$ となる。一方, 因 4 亿示すように，

$$
\begin{aligned}
& F_{1} \nsubseteq \psi\left(\mathrm{x}^{*}\right)+\left(\left(\diamond R_{1}^{1} \cap \diamond R_{2}^{1}\right) \cup \operatorname{Int} \square R_{1}^{1} \cup \operatorname{Int} \square R_{2}^{1}\right) \\
& F_{1} \nsubseteq \psi\left(\mathbf{x}^{*}\right)+\left(\left(\square R_{1}^{1} \cap \square R_{2}^{1}\right) \cup \operatorname{Int} \diamond R_{1}^{1} \cup \operatorname{Int} \diamond R_{2}^{1}\right)
\end{aligned}
$$

となるので, 弱意非支配必然解でも，強意非支配可能解でもない，したがって、非支配必然解で もない。
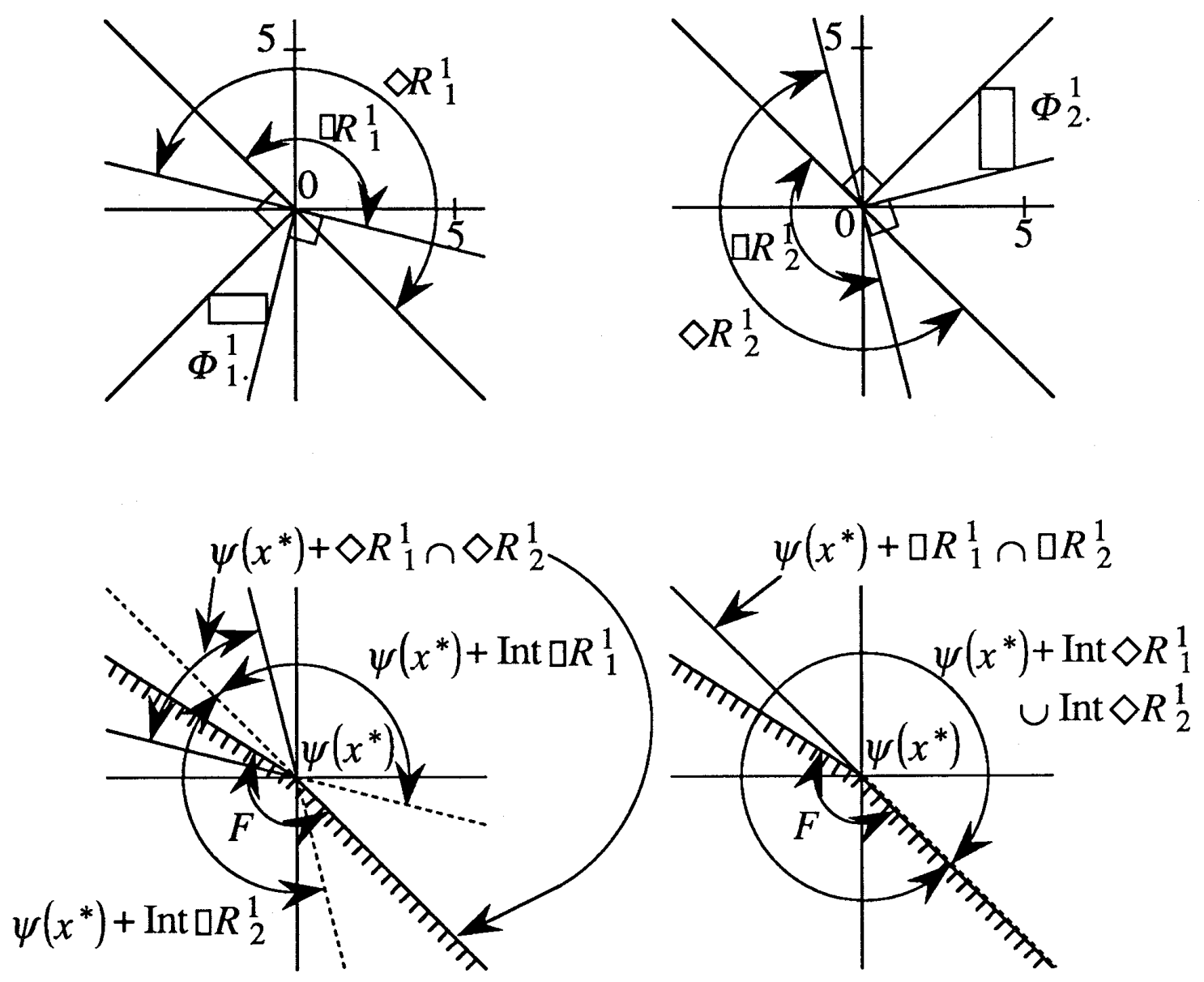

図 4 例 3.2 の図

(例 3.3) $\ell_{11}=2, u_{11}=4, \ell_{12}=-2, u_{12}=-1, \ell_{13}=u_{13}=\ell_{14}=u_{14}=0, \ell_{21}=2, u_{21}=$ $4, \ell_{22}=2, u_{22}=4, \ell_{23}=u_{23}=\ell_{24}=u_{24}=0$ とすると, $\square R_{i}^{1}, \diamond R_{i}^{1}(i=1,2)$ は図 5 のよ 5 K なる. $\psi\left(\mathrm{x}^{*}\right) \in F_{1}\left(\mathrm{x}^{*} \in F\right)$ であり,図 5 より,

$$
F_{1} \subseteq \psi\left(\mathbf{x}^{*}\right)+\left(\left(\square R_{1}^{1} \cap \square R_{2}^{1}\right) \cup \operatorname{Int} \diamond R_{1}^{1} \cup \operatorname{Int} \diamond R_{2}^{1}\right)
$$


$F_{1} \nsubseteq \psi\left(\mathbf{x}^{*}\right)+\left(\left(\diamond R_{1}^{1} \cap \diamond R_{2}^{1}\right) \cup \operatorname{Int} \square R_{1}^{1} \cup \operatorname{Int} \square R_{2}^{1}\right)$

となるので, $\mathbf{x}^{*}$ は強意非支配可能解 (仓ND $\left.\left(\mathbf{x}^{*}\right)\right)$ であるが, 弱意非支配必然解ではない.
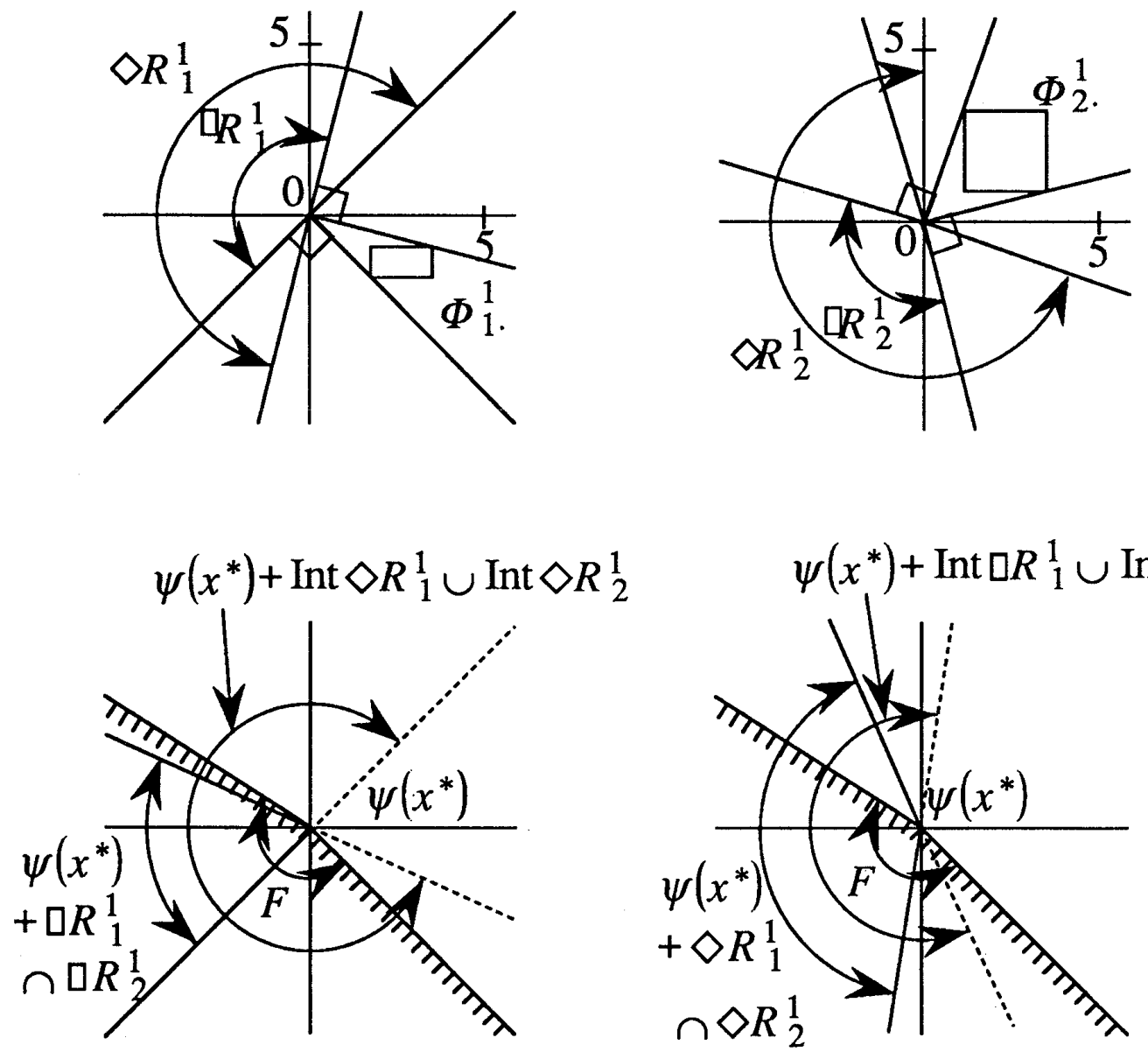

図 5 例 3.3 の図

(例 3.4) $\ell_{11}=2, u_{11}=3, \ell_{12}=1, u_{12}=2, \ell_{13}=u_{13}=\ell_{14}=u_{14}=0, \ell_{21}=4, u_{21}=$ $5, \ell_{22}=3, u_{22}=4, \ell_{23}=u_{23}=\ell_{24}=u_{24}=0$ とする.図 $6 \kappa, \square R_{i}^{1}, \diamond R_{i}^{1}(i=1,2)$ を示す. $\psi\left(\mathbf{x}^{*}\right) \in F_{1}\left(\mathbf{x}^{*} \in F\right)$ であり, 図 6 より,

$$
F_{1} \subseteq \psi\left(\mathbf{x}^{*}\right)+\left(\left(\diamond R_{1}^{1} \cap \diamond R_{2}^{1}\right) \cup \operatorname{Int} \square R_{1}^{1} \cup \operatorname{Int} \square R_{2}^{1}\right)
$$

$F_{1} \nsubseteq \psi\left(\mathbf{x}^{*}\right)+\left(\left(\square R_{1}^{1} \cap \square R_{2}^{1}\right) \cup \operatorname{Int} \diamond R_{1}^{1} \cup \operatorname{Int} \diamond R_{2}^{1}\right)$

となるので, $\mathbf{x}^{*}$ は弱意非支配必然解 (四 $\left.N D\left(\mathbf{x}^{*}\right)\right)$ であるが, 強意非支配可能解ではない. 

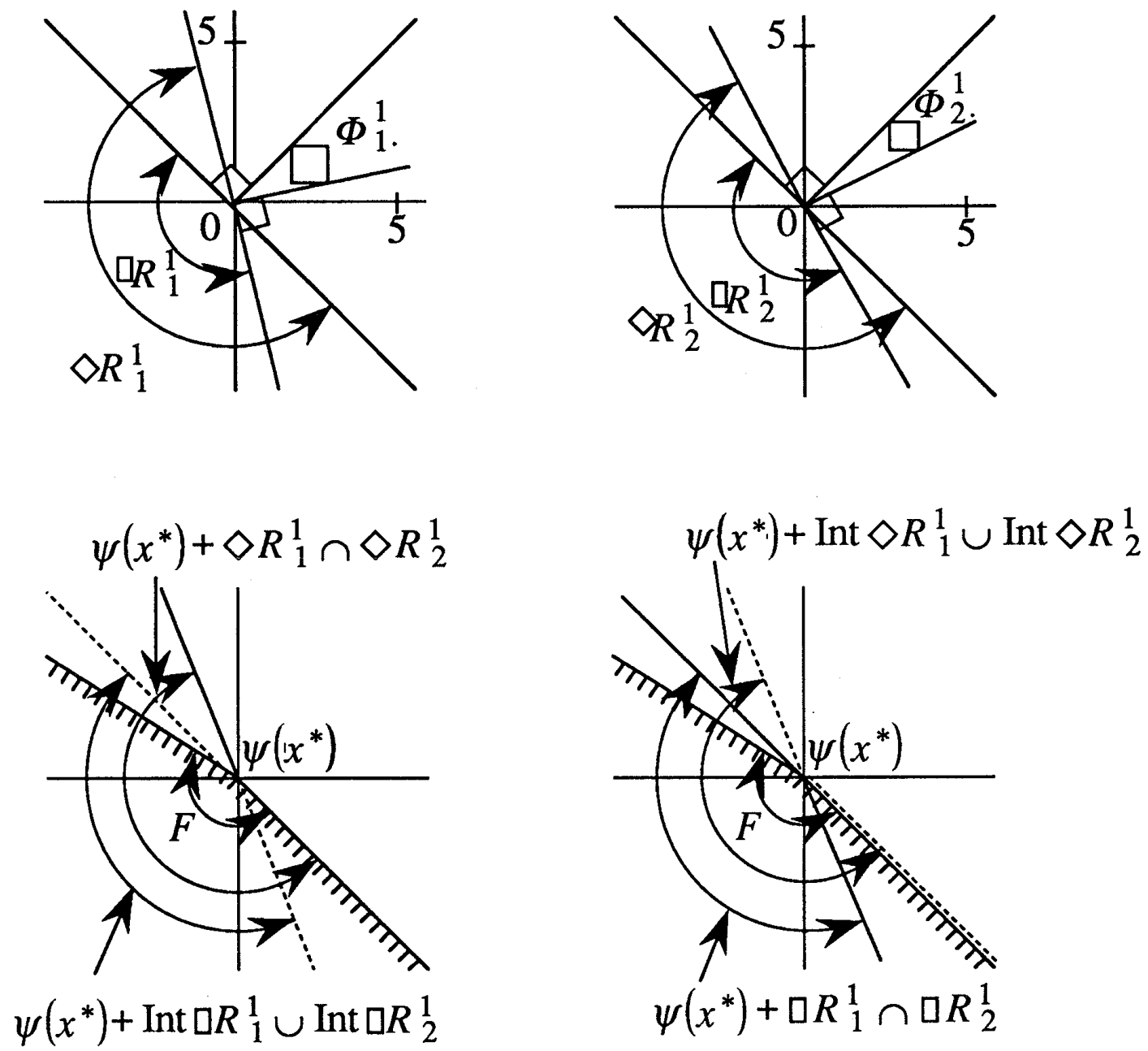

困 6 例 3.4 の困

(例 3.5) $\ell_{11}=1, u_{11}=3, \ell_{12}=2, u_{12}=3, \ell_{13}=u_{13}=\ell_{14}=u_{14}=0, \ell_{21}=4, u_{21}=5, \ell_{22}=$ $3, u_{22}=5, \ell_{23}=u_{23}=\ell_{24}=u_{24}=0$ とすると, $\square R_{i}^{1}, \diamond R_{i}^{1}(i=1,2)$ は図 7 のよらКなる. $\psi\left(\mathbf{x}^{*}\right) \in F_{1}\left(\mathbf{x}^{*} \in F\right)$ であり,図 7 より,

$$
\begin{aligned}
& F_{1} \subseteq \psi\left(\mathbf{x}^{*}\right)+\left(\left(\diamond R_{1}^{1} \cap \diamond R_{2}^{1}\right) \cup \operatorname{Int} \square R_{1}^{1} \cup \operatorname{Int} \square R_{2}^{1}\right) \\
& F_{1} \subseteq \psi\left(\mathbf{x}^{*}\right)+\left(\left(\square R_{1}^{1} \cap \square R_{2}^{1}\right) \cup \operatorname{Int} \diamond R_{1}^{1} \cup \operatorname{Int} \diamond R_{2}^{1}\right) \\
& F_{1} \nsubseteq \psi\left(\mathbf{x}^{*}\right)+\left(\left(\square R_{1}^{1} \cap \square R_{2}^{1}\right) \cup \operatorname{Int} \square R_{1}^{1} \cup \operatorname{Int} \square R_{2}^{1}\right)
\end{aligned}
$$

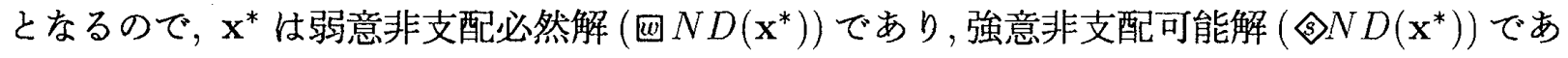
るが, 非支配必然解ではない。 

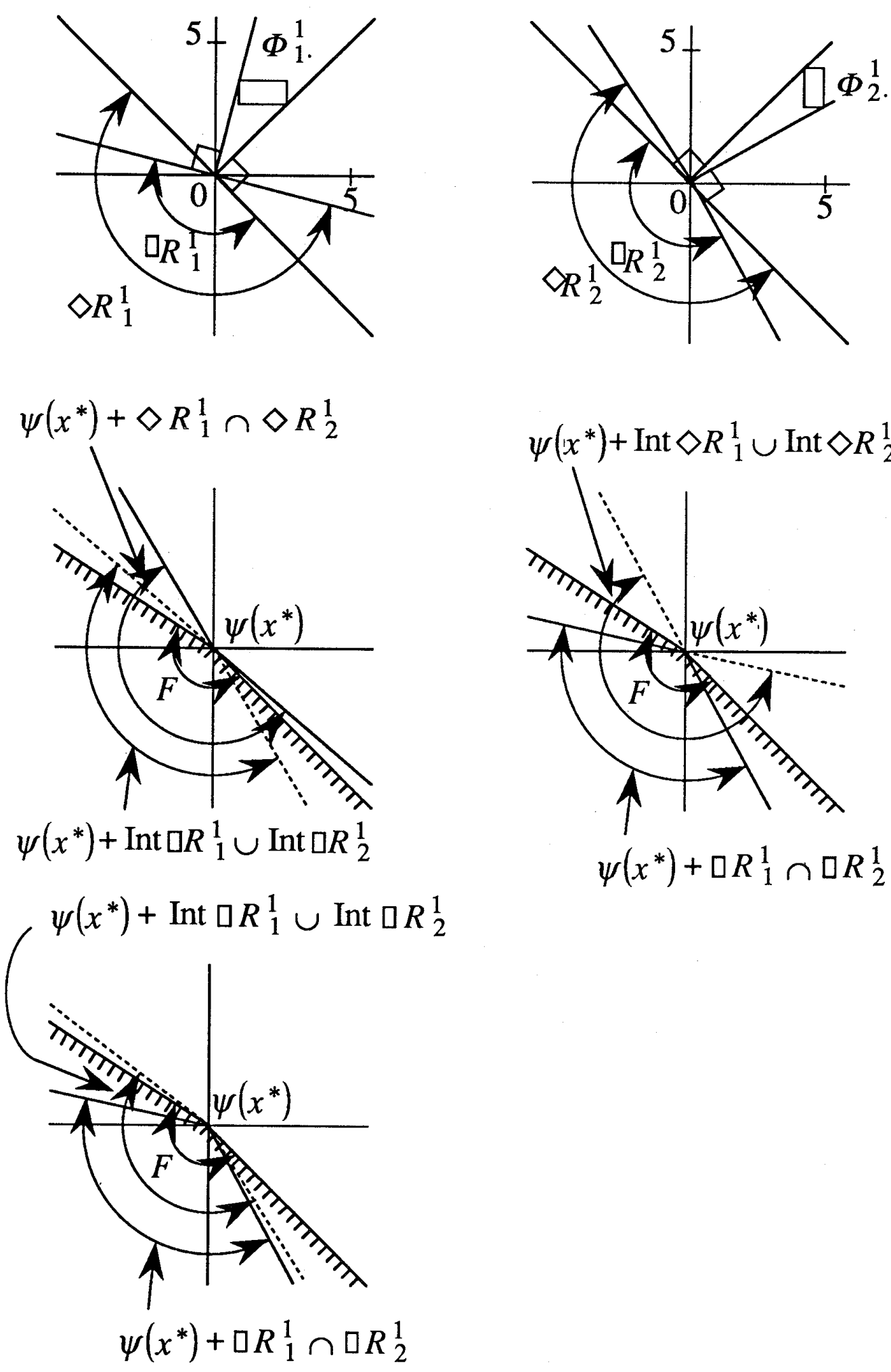

$\psi\left(x^{*}\right)+\operatorname{Int} \diamond R_{1}^{1} \cup \operatorname{Int} \diamond R_{2}^{1}$

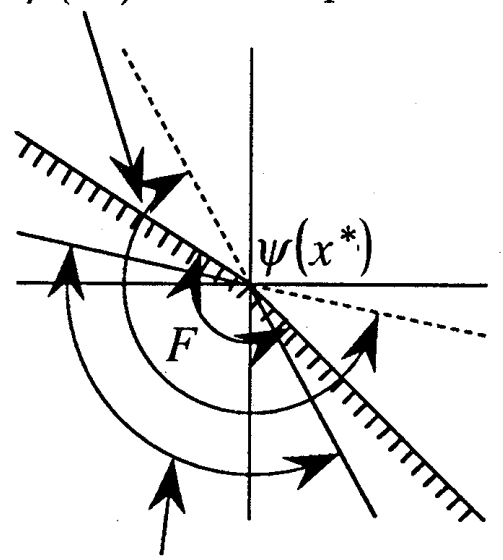

$\psi\left(x^{*}\right)+\square R_{1}^{1} \cap \square R_{2}^{1}$

図7例 3.5 の図 


\section{Bitran の二つの有効解との関係}

Bitran [2] は，区間多目的線形計画問題 (2.4) 式に対し,二つの有効解が考えられるととを指 摘している.それらは, 次のよ5に定義される ${ }^{7}$.

[定義 7] (必然的有効解，可能的有効解)

区間多目的線形計画問題 (2.4) 式に対し, 次の二つの有効解が定義できる.

i） $\mathbf{x}^{*}$ が必然的有効解であるととを $\square E F\left(\mathbf{x}^{*}\right)$ と記し，次のよら飞定義する.

$$
\begin{aligned}
& \square E F\left(\mathbf{x}^{*}\right) \triangleq \mathbf{x}^{*} \in F \\
& \quad \wedge \forall C\left(C \in \Phi \rightarrow \sim \exists \mathbf{x}\left(\mathbf{x} \in F \wedge D^{s}\left(C \mathbf{x}, C \mathbf{x}^{*}\right)\right)\right)
\end{aligned}
$$

ii) $\mathbf{x}^{*}$ が可能的有効解であるととを $\diamond E F\left(\mathbf{x}^{*}\right)$ と記し，次のように定義する.

$$
\begin{aligned}
& \diamond E F\left(\mathbf{x}^{*}\right) \triangleq \mathbf{x}^{*} \in F \\
& \wedge \exists C\left(C \in \Phi \wedge \sim \exists \mathbf{x}\left(\mathbf{x} \in F \wedge D^{s}\left(C \mathbf{x}, C \mathbf{x}^{*}\right)\right)\right)
\end{aligned}
$$

必然的有効解は， $C \in \Phi$ なるすへてての $C$ について有効となる解であり,区間を係数のとり 万る範囲と考えれば, 必然的に有効な解と考えるととができる。ての意味において，本研究では， 必然的有効解とい5. \&た, 可能的有效解は, $C \in \Phi$ なる少なくとも一つの $C$ について有効々な る解であり, 可能的に有効な解と考えるととができる.

(例 4.1) $\Phi, F$ が有限個の要素をもつ次の場合を考える.

$$
\begin{gathered}
\Phi=\left\{C^{1}, C^{2}, C^{3}\right\}, F=\left\{\mathbf{x}^{1}, \mathbf{x}^{2}, \mathbf{x}^{3}\right\} \\
C^{1}=\left(\begin{array}{cc}
-3 & -3 \\
4 & 4
\end{array}\right), C^{2}=\left(\begin{array}{cc}
-3 & -3 \\
5 & 3
\end{array}\right), C^{3}=\left(\begin{array}{cc}
-2 & -4 \\
4 & 4
\end{array}\right) \\
\mathbf{x}^{1}=(3,4)^{t}, \mathbf{x}^{2}=(4.2)^{t}, \mathbf{x}^{3}=(7,0)^{t}
\end{gathered}
$$

目的関数の各係数行列が実現したときの各解に対する目的関数の值を表 3 に示す.表 3 より,す べての $C^{i} \in \Phi(i=1,2,3)$ につ々て, $C^{i} \mathbf{x}^{j} \geqq C^{i} \mathbf{x}^{3}$ かつ $C^{i} \mathbf{x}^{3} \geq C^{i} \mathbf{x}^{j}$ すなわち, $D^{s}\left(C^{i} \mathbf{x}^{j}\right.$, $\left.C^{i} \mathbf{x}^{4}\right)$ が成立する $\mathbf{x}^{j} \in F$ が存在しないので, $\mathbf{x}^{4} \in F$ は必然的有効解 $\left(\square E F\left(\mathbf{x}^{4}\right)\right)$ である.

\begin{tabular}{|c|c|c|c|}
\hline 解 係数行列 & $C^{1}$ & $C^{2}$ & $C^{3}$ \\
\hline $\mathbf{x}^{1}$ & $(-21,28)^{t}$ & $(-21,27)^{t}$ & $(-22,28)^{t}$ \\
\hline$x^{2}$ & $(-18,24)^{t}$ & $(-18,26)^{t}$ & $(-16,24)^{t}$ \\
\hline $\mathbf{x}^{3}$ & $(-21,28)^{t}$ & $(-21,35)^{t}$ & $(-14,28)^{t}$ \\
\hline
\end{tabular}
$\mathbf{x}^{4} \in F$ は可能的有効解でもある. をた, $C^{1} \in \Phi$ につ々て, $C^{1} \mathbf{x}^{j} \geqq C^{1} \mathbf{x}^{2}$ かつ $C^{1} \mathbf{x}^{2} \geq C^{1} \mathbf{x}^{j}$ 寸なわち, $D^{s}\left(C^{1} \mathbf{x}^{j}, C^{1} \mathbf{x}^{2}\right)$ が成立する $\mathbf{x}^{j} \in F$ が存在しないので, $\mathbf{x}^{2} \in F$ 梳可能的有効解 $\left(\diamond E F\left(\mathbf{x}^{2}\right)\right)$ である.しかし, $C^{3} \in \Phi$ について, $C^{3} \mathbf{x}^{3} \geqq C^{3} \mathbf{x}^{2}$ かつ $C^{3} \mathbf{x}^{2} \geqq C^{3} \mathbf{x}^{3}$ すなわち, $D^{s}\left(C^{3} \mathbf{x}^{3}, C^{3} \mathbf{x}^{2}\right)$ が成立し, $\mathbf{x}^{3} \in F$ であるので, $\mathbf{x}^{2}$ は必然的有効解ではない. 同様に, $\mathbf{x}^{1}$ 法可能 的有効解であるが，必然的有効解ではない。

表 3. 例 4.1 Kおける各解の目的関数の值

以下では, Bitran の必然的有効解, 可能的有効解がそれぞれ, 本研究の非支配必然解, 非支配 
可能解に一致するととを示す.まず, 必要となる補題をいくつか証明する.

[補題 1] $S^{i}, i=1,2, \cdots, k, T, G$ を与えられた行列とし, $S^{i}, i=1,2, \cdots, k$ は空でないとする. てのとき, 次の [I], [II] のいずれかが成立する。しかし，同時に成立するととはない。

[I] 次式反解 $\mu$ が存在する.

$$
\left\{\begin{array}{l}
S^{i} \boldsymbol{\mu} \geq 0, i=1,2, \cdots, k \\
T \boldsymbol{\mu} \geqq 0 \\
G \boldsymbol{\mu}=0
\end{array}\right.
$$

[II] 次式に解 $\left(\mathbf{z}_{1}, \mathbf{z}_{2}, \cdots, \mathbf{z}_{k}, \mathbf{w}, \mathbf{v}\right)$ が存在する.

$$
\left\{\begin{array}{l}
\sum_{i=1}^{k} \mathbf{z}_{i} S^{i}+\mathbf{w} T+\mathbf{v} G=0 \\
\mathbf{z}_{1}>0 \text { または } \mathbf{z}_{2}>0 \text { または } \cdots \text { または } \mathbf{z}_{k}>0 \\
\mathbf{z}_{i} \geqq 0, i=1,2, \cdots, k, \mathbf{w} \geqq 0
\end{array}\right.
$$

証明は, 文献 [16]の二者択一の定理と同様に証明できるので, 省略する。

[補題 2] $\mathrm{x}^{*} \in F$ Kつレて, $D E F\left(\mathbf{x}^{*}\right)$ が成立するための必要十分条件は, 次式を満をす解 $(\mathbf{z}$, $\mathbf{w}, \mathbf{v}, \mathbf{h})$ が存在するととである.

$$
\left\{\begin{array}{l}
\mathbf{h}+\mathbf{w} E+\mathbf{v} A=0 \\
\mathbf{z} L \leqq \mathbf{h} \leqq \mathbf{z} U \\
\mathbf{z}>0, \mathbf{w} \geqq 0
\end{array}\right.
$$

ただし,$E$ は $n \times n$ の対角行列で, $\mathbf{x}^{*}=\left(x_{1}^{*}, x_{2}^{*}, \cdots, x_{n}^{*}\right)^{t}$ とし, $e_{j j}$ を $E$ の $(j, j)$ 成分とすると,

$$
e_{j j} \triangleq\left\{\begin{array}{l}
1 ; x_{j}^{*}=0 \\
0 ; x_{j}^{*}>0
\end{array}\right.
$$

と定義される.

(証明) 定義 7 および文献 [7] の系 1.3 より， $x^{*} \in F K つ \curvearrowleft て \diamond E F\left(\mathbf{x}^{*}\right)$ が成立するための必 要十分条件は,

$$
\left\{\begin{array}{l}
\mathbf{z} C+\mathbf{w} E+\mathbf{v} A=0 \\
\mathbf{z}>0, \mathbf{w} \geqq 0
\end{array}\right.
$$

飞解 $(\mathbf{z}, \mathbf{w}, \mathbf{v})$ が存在するような $C \in \Phi$ が存在するととである.とのととと, $\mathbf{z}>0$ より,任意の $C \in \Phi$ kつ々て, $\mathbf{z} L \leqq \mathbf{z} C \leqq \mathbf{z} U$ が成立し, $\mathbf{z} L \leqq \mathbf{h} \leqq \mathbf{z} U$ なる任意の $\mathbf{h}$ Кつ々て, $\mathbf{h}=\mathbf{z} C$ な るC$C \Phi$ が存在することにより,明らかである.

(証明終)

[補題 3] $2^{n}$ 個の $C^{i} \in M, i=1,2, \cdots, 2^{n}$ を $\cup_{i=1}^{2^{n}}\left\{C^{i}\right\}=M$ なる $p \times n$ 行列とし,

$$
\begin{aligned}
& I_{L}^{j}=\left\{i / C_{\cdot j}^{i}=L_{\cdot j}\right\}, j=1,2, \cdots, n \\
& I_{U}^{j}=\left\{i / C_{\cdot j}^{i}=U_{\cdot j}\right\}, j=1,2, \cdots, n
\end{aligned}
$$




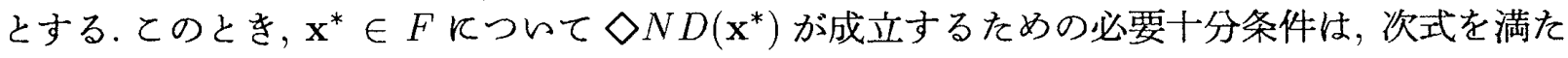
す解 $\left(\mathbf{z}_{1} \mathbf{z}_{2}, \cdots, \mathbf{z}_{2^{n}}, \mathbf{w}, \mathbf{v}\right)$ が存在することである.

$$
\left\{\begin{array}{l}
\left(\sum_{i \in I_{L}^{j}} \mathbf{z}_{i}\right) L \cdot j+\left(\sum_{i \in I_{U}^{j}} \mathbf{z}_{i}\right) U_{\cdot j} \\
\quad+\mathbf{w} E_{\cdot j}+\mathbf{v} A \cdot j=0, j=1,2, \cdots, n \\
\mathbf{z}_{1}>0 \text { まをは } \mathbf{z}_{2}>0 \text { または } \cdots \text { または } \mathbf{z}_{2^{n}>0}>0, \quad \mathbf{z}_{i} \geqq 0, i=1,2, \cdots, 2^{n}, \mathbf{w} \geqq 0
\end{array}\right.
$$

(証明) 定義 4,6 および命題 4 より, $\mathbf{x}^{*} \in F \ltimes つ ん て \diamond N D\left(\mathbf{x}^{*}\right)$ が成立するための必要十分 条件は,

$$
\left\{\begin{array}{l}
C^{i} \boldsymbol{\mu} \geq 0, i=1,2, \cdots, 2^{n} \\
E \boldsymbol{\mu} \geqq 0 \\
A \boldsymbol{\mu}=0
\end{array}\right.
$$

に解 $\boldsymbol{\mu か ゙}$ 存在しないととである.補題 1 より,ての条件は，

$$
\left\{\begin{array}{l}
\sum_{i=1}^{2^{n}} \mathbf{z}_{i} C^{i}+\mathbf{w} E+\mathbf{v} A=0 \\
\mathbf{z}_{1}>0 \text { または } \mathbf{z}_{2}>0 \text { または } \cdots \text { または } \mathbf{z}_{2^{n}}>0 \\
\mathbf{z}_{i} \geqq 0, i=1,2, \cdots, 2^{n}, \mathbf{w} \geqq 0
\end{array}\right.
$$

飞解 $\left(\mathbf{z}_{1}, \mathbf{z}_{2}, \cdots, \mathbf{z}_{2^{n}}, \mathbf{w}, \mathbf{v}\right)$ が存在するという条件と等価になる.さらに, 任意の $i \in\{1,2, \cdots$, $\left.2^{n}\right\}$ につて,$C_{\cdot j}^{i}=L_{. j}$ あるいは $C_{\cdot j}^{i}=U_{\cdot j}$ が成立するととに注意すると，ての式は (4.9) 式に なる。

(証明終)

補題 2,3 より, 次の命題が成立する.

[命題 10] 次式が成立する.

$$
\begin{aligned}
& \square E F\left(\mathbf{x}^{*}\right) \Leftrightarrow \square N D\left(\mathbf{x}^{*}\right) \\
& \diamond E F\left(\mathbf{x}^{*}\right) \Leftrightarrow \diamond N D\left(\mathbf{x}^{*}\right)
\end{aligned}
$$

(証明) 定義 7 より，

$$
\begin{aligned}
& \square E F\left(\mathbf{x}^{*}\right)=\mathbf{x}^{*} \in F \\
& \wedge \forall C \forall \mathbf{x}\left(C \in \Phi \wedge \mathbf{x} \in F \rightarrow \sim D^{s}\left(C \mathbf{x}, C \mathbf{x}^{*}\right)\right) \\
& \diamond E F\left(\mathbf{x}^{*}\right)=\mathbf{x}^{*} \in F \\
& \wedge \exists C \forall \mathbf{x}\left(C \in \Phi \wedge\left(\mathbf{x} \in F \rightarrow \sim D^{s}\left(C \mathbf{x}, C \mathbf{x}^{*}\right)\right)\right)
\end{aligned}
$$


と表せる。をた，定義 $4,6 よ り ，$

$$
\begin{aligned}
\square & N D\left(\mathbf{x}^{*}\right)=\mathbf{x}^{*} \in F \\
& \wedge \exists \mathbf{x}\left(\mathbf{x} \in F \wedge \exists C\left(C \in \Phi \wedge D^{s}\left(C \mathbf{x}, C \mathbf{x}^{*}\right)\right)\right) \\
= & \mathbf{x}^{*} \in F \\
& \wedge \forall \mathbf{x} \forall C\left(\mathbf{x} \in F \wedge C \in \Phi \rightarrow \sim D^{s}\left(C \mathbf{x}, C \mathbf{x}^{*}\right)\right) \\
\diamond & N D\left(\mathbf{x}^{*}\right)=\mathbf{x}^{*} \in F \\
& \wedge \sim \exists \mathbf{x}\left(\mathbf{x} \in F \wedge \forall C\left(C \in \Phi \rightarrow D^{s}\left(C \mathbf{x}, C \mathbf{x}^{*}\right)\right)\right) \\
= & \mathbf{x}^{*} \in F \\
& \wedge \forall \mathbf{x} \exists C\left(\mathbf{x} \in F \rightarrow C \in \Phi \wedge \sim D^{s}\left(C \mathbf{x}, C \mathbf{x}^{*}\right)\right)
\end{aligned}
$$

となる。したがって, (4.10)式は明らかである。また，

$$
\begin{aligned}
& \exists C \forall \mathbf{x}\left(C \in \Phi \wedge\left(\mathbf{x} \in F \rightarrow \sim D^{s}\left(C \mathbf{x}, C \mathbf{x}^{*}\right)\right)\right) \\
& \quad \Rightarrow \forall \mathbf{x} \exists C\left(C \in \Phi \wedge\left(\mathbf{x} \in F \rightarrow \sim D^{s}\left(C \mathbf{x}, C \mathbf{x}^{*}\right)\right)\right) \\
& C \in \Phi \wedge\left(\mathbf{x} \in F \rightarrow \sim D^{s}\left(C \mathbf{x}, C \mathbf{x}^{*}\right)\right) \\
& \quad \Rightarrow \mathbf{x} \in F \rightarrow C \in \Phi \wedge \sim D^{s}\left(C \mathbf{x}, C \mathbf{x}^{*}\right)
\end{aligned}
$$

が成立するととより, $\diamond E F\left(\mathbf{x}^{*}\right) \Rightarrow \diamond N D\left(\mathbf{x}^{*}\right)$ が成立する.ゆえK, $\diamond N D\left(\mathbf{x}^{*}\right) \Rightarrow \diamond E F\left(\mathbf{x}^{*}\right)$ を示せばよい. 補題 2,3 より，(4.9) 式に解が存在するとを，(4.5) 式に解が存在するととを証明 すればよい. (4.9) 式に解 $\left(\mathbf{z}_{1}^{*}, \mathbf{z}_{2}^{*}, \cdots, \mathbf{z}_{2^{n}}^{*}, \mathbf{w}^{*}, \mathbf{v}^{*}\right)$ が存在すると仮定する.とのとき，

$$
\begin{aligned}
& \mathbf{h}^{*} \triangleq\left(h_{1}^{*}, h_{2}^{*}, \cdots, h_{n}^{*}\right) \\
& h_{j}^{*} \triangleq\left(\sum_{i \in I_{L}^{j}} \mathbf{z}_{i}^{*}\right) L_{\cdot j}+\left(\sum_{i \in I_{U}^{j}} \mathbf{z}_{i}^{*}\right) U_{\cdot j}, j=1,2, \cdots, n \\
& \mathbf{z}^{*} \triangleq \sum_{i=1}^{2^{n}} \mathbf{z}_{i}^{*}
\end{aligned}
$$

と定義すると, 明らかに，

$$
\left\{\begin{array}{l}
\mathbf{h}^{*}+\mathbf{w}^{*} E+\mathbf{v}^{*} A=0 \\
\mathbf{z}^{*} L \leqq \mathbf{h}^{*} \leqq \mathbf{z}^{*} U \\
\mathbf{z}^{*}>0, \mathbf{w}^{*} \geqq 0
\end{array}\right.
$$

が成立し, $\left(\mathbf{z}^{*}, \mathbf{w}^{*}, \mathbf{v}^{*}, \mathbf{h}^{*}\right)$ は (4.5) 式の解となる。

(証明終)

補題 2,3 を証明するために，区間で表された $\Phi$ 各成分間に相互関係がないととを暗に用 いているので，ての条件が成立しない場合には，必ずしも(4.11) 式が成立するとは限らない。し かし, (4.10) 式, $\diamond E F\left(\mathbf{x}^{*}\right) \Rightarrow \diamond N D\left(\mathbf{x}^{*}\right)$ は常に成立する.

命題 10 は, Bitranの二つの有効解が本研究の非支配必然解, 非支配可能解に一致するとと を示している。

\section{5. おわりに}

区間多目的線形計画問題に対し, 支配関係を可能, 必然などの様相概念を用いて構成すると とにより，四つの非支配解を定義した。 また，四つの非支配解の関係および性質を調へ，Bitran による二つの有効解との関係を明らかにした。 
本研究では，支配錐が非正象限である支配関係につんて非支配解を定義したが，その他の支 配関係につんても同様に定義できる. \&た，多目的非線形計画問題に対しても, 本研究の非支配 解の概念を適用するてとができる.係数がファジイ数で制限される多目的計画問題についても， 存在記号 $\exists$ sup $\nwarrow$, 全称記号 $\forall を \inf K$, 連言 $\wedge$ min 演算 $K$, 含意 $\rightarrow$ Dienes 含意飞置を 換え，可能性測度，必然性測度 [6] [11] を用いて拡張できる.さらに，区間で制限された係数間に 相互関係がある場合につレても,議論する必要がある。てれらについての詳細な論究は, 今後の 課題である。

脚注

1) 類似したものとして，一般化線形計画問題 [5] [29] があるが，一般化線形計画問題では, 係数べクトルの凸集合は係数間の関係を表し,係数べクトルも決定変数として扱われて らる.したがって，係数ベクトルの凸集合が不明確さを表す Inexact 線形計画問題とは， 考え方が異なる.しかし，一般化線形計画法による解は, Inexact 線形計画問題の楽観的 な解と考えられる。

2) とてでは, 最大化問題を考えている.最小化問題の場合には, 非負象限となる.

3) ある係数のとりらる範囲 (区間) が他の係数のとる值に依存しないととを相互関係がな レという [38].

4) 式変形については, たとえば, 文献 [18]などを参照されたい.

5)とのてとより，新をに非反射的な支配関係として，

$$
\bigcap^{s} D\left(\mathbf{x}^{1}, \mathbf{x}^{2}\right) \triangleq \diamond^{s} D\left(\mathbf{x}^{1}, \mathbf{x}^{2}\right) \vee \varpi^{s} D\left(\mathbf{x}^{1}, \mathbf{x}^{2}\right)
$$

を考えるとともできる．

6) こてでは, (2.28) 式が成立するので, 四つであるが, (2.28) 式が成立しない場合は五つに なる.また，脚注 5 の支配関係 $\bigcup^{s} D$ を用いて，定義 6 と同様に，

$$
\oint N D\left(\mathbf{x}^{*}\right) \triangleq \sim \exists \mathbf{x}\left(\mathbf{x} \in F \wedge \oint^{s} D\left(\mathbf{x}, \mathbf{x}^{*}\right)\right)
$$

を定義できるが，

$$
\bigcap N D\left(\mathbf{x}^{*}\right) \Leftrightarrow \varpi N D\left(\mathbf{x}^{*}\right) \wedge \diamond N D\left(\mathbf{x}^{*}\right)
$$

が成立するため, $0 N D\left(\mathbf{x}^{*}\right)$ は考えないてとにする。

7）Bitran [2] は集合を用いて定義しているが，とてでは,述語を用いて記述する.

\section{参考文献}

[1] Bard, J.F.: Inexact Linear Programming with Generalized Technological Matrix Sets. European Journal of Operational Research, Vol. 16 (1984), 107-112.

[2] Bitran, G.R.: Linear Multiple Objective Problems with Interval Coefficients. Management Science, Vol. 26, No. 7 (1980), 694-706.

[3] Buckley, J.J.: Possibility and Necessity in Optimization.Fuzzy Sets and Systems, Vol. 25 (1988), 1-13.

[4] Buckley, J.J.: Possibilistic Linear Programming with Triangular Fuzzy Numbers. Fuzzy Sets and Systems, Vol. 26 (1988), 135-138. 
[5] Dantzig, G.B.: Linear Programming and Extensions. Princeton University Press, Princeton, 1963. 小山昭雄訳: 線型計画法とその周辺. ホルト・サウンダース・ジャパ ン, 1983.

[6] Dubois, D.: Linear Programming with Fuzzy Data. Analysis of Fuzzy Information: Vol. III Applications in Engineering and Science (ed. J.C. Bezek). CRC Press, Boca Raton, Florida, 1987, 241-263.

[7] Evans, J.P. and Steuer, R.E.: A Revised Simplex Method for Linear Multiple Objective Programs. Mathematical Programming, Vol. 5 (1973), 54-72.

[8] Falk, J.E.: Exact Solutions of Inexact Linear Programs. Operations Research, Vol. 24 (1976), 783-787.

[9] Gal, T. and Nedoma, J.: Multiparametric Linear Programming. Management Science, Vol. 18, No. 7 (1972), 406-422.

[10] Hartley, R.: Validity of Local Search in Multiparametric and Vector Linear Programming. Journal of the Operational Research Society, Vol. 39, No. 1 (1988), 83-90.

[11] 乾口雅弘, 市橋秀友, 田中英夫: 様相概念に基づく意思决定方式とそのファジィ多目的 線形計画問題一の応用. Journal of the Operations Research Society of Japan, Vol. 30, No. 4 (1987), 449-470.

[12] 乾口雅弘, 市橋秀友, 田中英夫: 多属性価值関数を用いたファジィ線形計画法. Journal of the Operations Research Society of Japan, Vol. 31, No. 1 (1988), 121-142.

[13] 乾口雅弘, 久米靖文: 区間概念に基づく目標計画問題の四つの定式化. 日本経営工学会 誌, 39, 3 (1988), 146-152.

[14] Luhandjula, M.K.: On Possibilistic Linear Programming. Fuzzy Sets and Systems, Vol. 18 (1986), 15-30.

[15] Luhandjula, M.K.: Multiple Objective Programming Problems with Possibilistic Coefficients. Fuzzy Sets and Systems, Vol. 21 (1987), 135-145.

[16] Mangasarian, O.L.: Nonlinear Programming. McGraw-Hill Book Company, New York, 1969. 関根智明訳: 非線形計画法. 培風館, 1972.

[17] Moore, R.E.: Methods and Applications of Interval Analysis. siam, Philadelphia, 1979.

[18] 長尾真, 淵一博: 論理と意味. 岩波書店, 1983.

[19] Orlovsky, S.A.: On Formalization of a General Fuzzy Mathematical Problem. Fuzzy Sets and Systems, Vol. 3 (1980), 311-321.

[20] Orlovski, S.A.: Multiobjective Programming Problems with Fuzzy Parameters. Control and Cybernetics, Vol. 13, No. 3 (1984), 175-183.

[21] 坂和正敏: 線形システムの最適化. 森北出版, 1984.

[22] 坂和正敏: 多目的計画法とファジイ計画法. システムと制御, 32, 8 (1988), 447-454.

[23] 坂和正敏: ファジィ理論の基礎と応用. 森北出版, 1989.

[24] 坂和正敏, 矢野均: ファジィパラメータを含む多目的非線形計画問題に対する対話型意 思决定. 計測自動制御学会論文集, 22,2 (1986), 162-167.

[25] 坂和正敏, 矢野均: ファジーパラメータを含む多目的線形計画問題に対する統一的アプ ローチ.電子情報通信学会論文誌, J71-A, 8 (1988), 1569-1575.

[26] 坂和正敏, 矢野均: ファジーパラメータを含む多目的非線形計画問題に対する実行可能 性とパレート最適性. 電子情報通信学会論文誌, J-72-A, 8 (1989), 1062-1068. 
[27] 坂和正敏, 矢野均, 高橋淳也: ファジーパラメータを含む多目的線形計画問題に対する拡 張パレート最適性の概念とその性質. 電子情報通信学会論文誌, J72-A，4 (1989), 678684.

[28] 坂和正敏, 矢野均, 高橋淳也: ファジーパラメータを含む多目的線形分数計画問題に対 するパレート最適性. 電子情報通信学会論文誌, J72-A, 6 (1989), 931-937.

[29] 関根泰次: 数理計画法. 岩波畫店, 1976.

[30] Soyster, A.L.: Inexact Linear Programming with Generalized Resource Sets. European Journal of Operational Research, Vol. 3 (1979), 316-321.

[31] Steuer, R.E.: Algorithms for Linear Programming Problems with Interval Objective Function Coefficients. Mathematics of Operations Research, Vol. 6, No. 3 (1981), 331-348.

[32] Tanaka, H. and Asai, K.: Fuzzy Linear Programming with Fuzzy Numbers. Fuzzy Sets and Systems, Vol. 13 (1984), 1-10.

[33] 田中英夫, 市橋秀友, 浅居喜代治: ファジィ目的関数によるファジィ線形計画問題の定 式化. Journal of the Operations Research Society of Japan, Vol. 27, No. 2 (1984), 178-190.

[34] Van De Panne, C.: A Node Method for Multiparametric Linear Programming. Management Science, Vol. 21, No. 9 (1975), 1014-1020.

[35] Wendell, R.E.: The Tolerance Approach to Sensitivity Analysis in Linear Programming. Management Science, Vol. 31, No. 5 (1985), 564-578.

[36] Yu, P.L.: Cone Convexity, Cone Extreme Points, and Nondominated Solutions in Decision Problems with Multiobjectives. Journal of Optimization Theory and Applications, Vol. 14, No. 3 (1974), 319-377.

[37] Yu, P.L. and Zeleny, M.: Linear Multiparametric Programming by Multicriteria Simplex Method. Management Science, Vol. 23, No. 2 (1976), 159-170.

[38] Zadeh, L.A.: Fuzzy Sets as a Basis for a Theory of Possibility. Fuzzy Sets and Systems, Vol. 1 (1978), 3-28.

連絡先:

乾口雅弘

堺市百舌鳥梅町 4 丁 804

大阪府立大学工学部経営工学科 


\title{
ABSTRACT On the Concepts of Nondominated Solutions for Multiobjective
Linear Programming Problems with Interval Coefficients
}

\author{
Masahiro Inuiguchi Yasufumi Kume \\ University of Osaka Prefecture
}

The coefficients of linear programming problems have been assumed to be determined as crisp values by the experts. However, the knowledge of the experts is not always so accurate to determine them as crisp values. The experts often have vague or imprecise knowledge of the coefficients. In such cases, it is not sufficient to fix the coefficients definitely. It is more sufficient to determine them only as regions in which the coefficients possibly take. From this point of view, the inexact linear programming, the interval linear programming and possibilistic linear programming have been proposed. In multiobjective linear programming, the ones with interval coefficients or with fuzzy coefficients have been proposed and the concept of efficiency is extended.

Bitran directly extended the efficiency to multiobjective linear programming problem with interval objective functions:

$$
\operatorname{Max}\left\{\Phi \mathbf{x} / \mathbf{x} \in \mathbf{R}^{n}, A \mathbf{x}=\mathbf{b}, \mathbf{x} \geqq 0\right\}
$$

where $A$ is an $m$ by $n$ matrix, $\mathbf{b}$ and $\mathbf{x}$ are respectively $m$ and $n$ vectors. $\Phi$ is a set of $p$ by $n$ matrices with components $c_{i j}$ in the interval $\left[\ell_{i j}, u_{i j}\right], i=1,2, \cdots, p$ and $j=1,2, \cdots, n$. By Bitran, it was pointed out that two kinds of efficient solutions can be considered.

In this paper, the solution concepts for the interval multiobjective linear program are proposed in a different manner from Bitran's approach. First, given a reflective domination relation in the objective space, a strong (nonreflective) domination relation is defined. The domination relations in the decision space are composed from the domination relations in the objective space, and using them the nonreflective domination relations are defined. The properties of nonreflective domination relations are investigated. Next, four concepts of nondominated solutions for the interval multiobjective linear programming problem, i.e. possibly nondominated solutions, necessarily nondominated solutions, strong possibly nondominated solutions and weak necessarily nondominated solutions, are defined using four nonreflective domination relations. The properties of these nondominated solutions are discussed and it is shown that an arbitrary element of each nondominated solution set can be expressed by a convex combination of the corresponding nondominated extreme points. Moreover, the relationships with Bitran's two efficient solutions are clarified. Two of four nondominated solutions are equivalent to Bitran's two efficient solutions and the others are intermediated ones between them. 